\title{
Pimiento (Capsicum annuum) cultivado bajo invernadero: correlaciones entre variables
}

\section{Sweet pepper (Capsicum annuum) grown under greenhouse conditions: correlations among variables}

\author{
Esteban Elizondo-Cabalceta ${ }^{1}$ \\ Universidad Nacional, Costa Rica \\ José Eladio Monge-Pérez ${ }^{2}$ \\ Universidad de Costa Rica, Costa Rica
}

\begin{abstract}
Resumen. Se establecieron correlaciones de Pearson para 27 genotipos de pimiento (incluyendo frutos de forma cónica, cuadrada y rectangular) producidos bajo invernadero, entre 12 variables cuantitativas, para conocer la asociación entre estos caracteres: altura de la planta $(\mathrm{m})$, área de la hoja ( $\mathrm{cm} 2)$, diámetro del tallo $(\mathrm{mm})$, longitud del tallo $(\mathrm{cm})$, ancho del fruto $(\mathrm{cm})$, longitud del fruto $(\mathrm{cm})$, espesor de la pared del fruto $(\mathrm{mm})$, número de frutos de primera calidad por planta, número de frutos totales por planta, edad al inicio de la cosecha (días después de trasplante), peso del fruto de primera calidad (g), y rendimiento comercial (ton/ha). El cultivo se manejó con fertirrigación, en sustrato de fibra de coco, y con poda española. Se presentaron 15 correlaciones de Pearson evaluadas en los 27 genotipos que fueron altas $(r \geq 0,69)$ y con significancia estadística $(p \leq 0,05)$, en cuyo caso se obtuvieron las regresiones lineales. Además, se obtuvieron otras seis correlaciones altas y con significancia estadística, según el tipo de pimiento (cónico o cuadrado). Se concluye que el tipo de pimiento incluido en el análisis, influye de manera importante en el resultado de las correlaciones.
\end{abstract}

Palabras clave. Capsicum annuum, calidad, rendimiento, correlación de Pearson, regresión lineal.

\begin{abstract}
For 27 sweet pepper genotypes (including conical, square and rectangular fruit shape) grown under greenhouse conditions the researchers estimated Pearson correlations among twelve quantitative variables, to know the association between these characters: plant height $(\mathrm{m})$, leaf area $(\mathrm{cm} 2)$, stem diameter $(\mathrm{mm})$, stem length $(\mathrm{cm})$, fruit width $(\mathrm{cm})$, fruit length $(\mathrm{cm})$, fruit wall thickness $(\mathrm{mm})$, number of first quality fruits per plant, number of total fruits per plant, number of days to the start of harvest (days after transplant), first quality fruit weight (g), and commercial yield (ton/ha). The crop was planted on coconut fiber as substrate, and managed with fertigation and Spanish pruning method. Among the 27 evaluated genotypes there were 15 Pearson correlations that were high $(r \geq 0,69)$ and statistically significant $(p \leq 0,05)$. In those cases the linear regression statistic were calculated. Statistically significant correlations were also observed in six additional cases, not across all genotypes but estimated according to the type of pepper (conical or square). It is concluded that the sweet pepper type included in the analysis has a large effect in the correlation results.
\end{abstract}

Keywords. Capsicum annuum, quality, yield, Pearson correlation, linear regression.

\footnotetext{
${ }^{1}$ Ingeniero agrónomo, Universidad Nacional, Costa Rica. Dirección electrónica: estebanec5@gmail.com

${ }^{2}$ Ingeniero agrónomo, Estación Experimental Agrícola Fabio Baudrit Moreno y Sede de Guanacaste, Universidad de Costa Rica, Costa Rica. Dirección electrónica: melonescr@yahoo.com.mx
} 


\section{Introducción}

El pimiento (Capsicum annuum L.) pertenece a la familia Solanaceae, y es un importante cultivo bajo invernadero en muchos países. En 2009, en Costa Rica existían alrededor de 41 hectáreas de pimiento y tomate cultivadas bajo ambientes protegidos (Marín, 2009). En este país, la mayoría de productores de pimiento (también llamado chile dulce en este país) utilizan sistemas de producción a campo abierto, alcanzando en 2006 un área de 285,8 ha (Jiménez, Campos, Vicente, Marín, Barrantes, \& Carrillo, 2007).

La horticultura protegida es una alternativa para los productores de Costa Rica. El uso de invernaderos se ha extendido alrededor del mundo con gran éxito (Wittwer \& Castilla, 1995); ellos permiten controlar mejor las variables de producción de los cultivos, como las condiciones climáticas, riego, fertilización, tutorado, poda, y el uso de sustratos para la siembra (Gil-Vásquez, Sánchez del Castillo, \& Miranda-Velásquez, 2003; Castilla, 2005; Marín, 2009).

La tecnología de producción en invernadero ha incrementado el rendimiento por área. Según unos autores, el rendimiento de pimiento en invernadero puede llegar a 80 ton/ha (Zúñiga-Estrada, Martínez-Hernández, Baca-Castillo, Martínez-Garza, Tirado-Torres, \& Kohashi-Shibata, 2004), mientras que, para otros investigadores, el mismo oscila entre 8,2 y 42,9 ton/ha a campo abierto, y entre 30,0 y 150,0 ton/ha bajo ambiente protegido (Jiménez, y otros, 2007).

En Costa Rica la variedad de pimiento más utilizada es el híbrido Nathalie F-1; aunque existen otros genotipos y tipologías del fruto con posibilidades de competir en el mercado nacional, que requiere principalmente frutos rojos de forma cónica, y en el mercado internacional, que demanda frutos de forma cuadrada o rectangular, y de colores anaranjado, amarillo y rojo (Jiménez, y otros, 2007).

El coeficiente de correlación es una herramienta estadística que generalmente muestra relaciones entre caracteres independientes, y el grado de relación lineal entre esas características. En la investigación en fitomejoramiento, por lo general se toman medidas de varios caracteres o variables a partir de la misma planta. Para determinar el grado y la dirección de las relaciones lineales que corresponden a esas mediciones realizadas, usualmente los investigadores utilizan el análisis de correlación simple (Cancaya, Balkaya, \& Karaagac, 2010).

La capacidad productiva en pimiento es una característica compleja, que resulta de varios componentes morfológicos: número de flores, tamaño del fruto, grosor de la pared del fruto, peso del fruto; todos estos caracteres son específicos de cada genotipo, y son altamente influenciados por las condiciones de crecimiento y desarrollo. El pimiento presenta una alta variabilidad de caracteres, y pocas correlaciones entre ellos. El establecimiento de correlaciones entre caracteres, y el grado de su influencia recíproca, es muy útil en los procesos de selección genética. El conocimiento de las correlaciones ofrece la posibilidad de evaluar los caracteres complejos mediante la evaluación de los caracteres menos complejos (Madosa, Ciulca, Velicevici, Avadanei, Sasu, Cioroga, \& Friskan, 2008).

En la India, unos investigadores encontraron que, en el caso del pimiento, la longitud del fruto, el ancho del fruto, y el número de frutos por planta, mostraron una correlación positiva significativa con el rendimiento (Sharma, Semwal, \& Uniyal, 2010). Otros investigadores también han realizado investigación en la correlación de variables en pimiento (Cancaya, y otros, 2010; Sasu, Madosa, Velicevici, Ciulca, Avadanei, \& Gorinoiu, 2013; Ben-Chaim \& Paran, 
2000; Madosa, y otros, 2008; Diwaker, Vijay, Rangare, \& Devi, 2012; Gupta, Daljeet, \& Ajay, 2009; Bharadwaj, Singh, \& Yadav, 2007; Baseerat, Khan, Mushtaq, Hussain, \& Nabi, 2013).

El objetivo de este trabajo fue establecer correlaciones de Pearson entre 12 variables cuantitativas para el cultivo de pimiento bajo ambiente protegido, en Alajuela, Costa Rica, para conocer la asociación entre estos caracteres.

\section{Materiales y métodos}

El ensayo se llevó a cabo en el invernadero de Hortalizas de la Estación Experimental Agrícola Fabio Baudrit Moreno de la Universidad de Costa Rica, situada en el Barrio San José de Alajuela. Este sitio está ubicado a una altitud de $883 \mathrm{msnm}$, y muestra una precipitación anual de
$1940 \mathrm{~mm}$ distribuidos de mayo a noviembre, y un promedio anual de temperatura de $22 \mathrm{oC}$.

El ensayo se llevó a cabo en un invernadero modelo XR de la marca Richel (Francia), tipo multicapilla, de plástico, con ventilación cenital automática. Se evaluaron 27 genotipos híbridos de pimiento (Capsicum annuum L.) (tabla 1), correspondientes a tres tipos, según la forma del fruto: cónico (12 genotipos), cuadrado (13 genotipos) y rectangular (2 genotipos).

La siembra del almácigo se realizó el día 7 de julio de 2010, y el trasplante se llevó a cabo el día 19 de agosto de 2010. El cultivo se sembró a una densidad de 2,60 plantas/m2, en sacos plásticos rellenos con fibra de coco como sustrato. El cultivo se manejó con fertirrigación, y se aplicó el sistema de poda española, que consistió en dejar las plantas a libre crecimiento. Se utilizaron

Tabla 1

Genotipos de pimiento utilizados en la investigación

\begin{tabular}{ll}
\hline Tipo de pimiento & Genotipos \\
\hline & Cortés (Distribuidora Agro Comercial); FBM-1 (Universidad de Costa \\
& Rica); FBM-2 (Universidad de Costa Rica); FBM-3 (Universidad de Costa \\
Cónico & Rica); FBM-7 (Universidad de Costa Rica); FBM-11 (Universidad de Costa \\
$(\mathrm{n}=12)$ & Rica); FBM-12 (Universidad de Costa Rica); Jumbo (Villaplants); Lamuyo \\
& Amarillo (Villaplants); Lamuyo Experimental (Villaplants); Tiquicia (Vi- \\
& llaplants); V-701 (Seracsa) \\
& Amarillo Americano (Villaplants); MACR-101-07 (Semillas Este Oeste); \\
& MACR-102-07 (Semillas Este Oeste); MACR-103-07 (Semillas Este Oeste); \\
Cuadrado & MACR-104-07 (Semillas Este Oeste); MACR-105-07 (Semillas Este Oeste); \\
$(\mathrm{n}=13)$ & Magno (Resusa); Oberon (Resusa); Rojo Americano (Villaplants); Sweet Pe- \\
& pper Red (Semillas Agrícolas de Costa Rica); Vikingo (Sakata); XPPAD-169 \\
& (Sakata); XPPAD-286 (Sakata) \\
Rectangular $(\mathrm{n}=2)$ & Estrella (Rauco);XC-425 (Seracsa) \\
\hline
\end{tabular}

Nota. Elaborado a partir de los genotipos utilizados. El nombre entre paréntesis corresponde a la empresa productora o comercializadora de la semilla de cada genotipo. 
goteros con una descarga de 2 litros/hora, y se utilizó un dosificador marca Dosatron, con una proporción de inyección de 1:64. Se aplicaron las sales solubles los días lunes, miércoles y viernes, a partir del primer día de trasplante; los días viernes se preparó una mezcla aumentada en un $50 \%$ con respecto a los otros días para nutrir al cultivo durante el fin de semana. Las sales aplicadas fueron: 17-5-19, nitrato de potasio, nitrato de calcio, fosfato monoamónico, y sulfato de magnesio. Asimismo, se hicieron aplicaciones foliares de los productos Fetrilon Combi ${ }^{\circledR}$, Manvert-Ca ${ }^{\circledR}$ y Kelpak ${ }^{\circledR}$, como fuentes de micronutrientes.

Para el combate de plagas y enfermedades, se estableció un sistema de monitoreo diario de la plantación, y con base en los datos de incidencia, se utilizó un manejo integrado de plagas, mediante trampas adhesivas (amarillas $\mathrm{y}$ azules), repelentes, productos biológicos (Bacillus thuringiensis, Beauveria bassiana, Metarhizium sp., Lecanicillium sp., Paecilomyces sp.), y plaguicidas sintéticos (Amistar ${ }^{\circledR} 50$ WG, Relámpago® 1,8 EC, Spintor ${ }^{\circledR} 12$ SC, Rally ${ }^{\circledR} 40$ WP, Bellis ${ }^{\circledR} 38$ WG, Cobrethane ${ }^{\circledR}$ 61,1 WP).
El inicio de la cosecha fue el día $1^{\circ}$ de noviembre de 2010, y la última cosecha se llevó a cabo el día 14 de abril de 2011. Los frutos se cosecharon en forma semanal, en el momento en que tenían una madurez mínima del 50 $\%$. Los frutos se clasificaron en tres categorías de calidad (primera, segunda y rechazo), de acuerdo a diversos parámetros, según la forma del pimiento (tablas 2 y 3 ).

A partir de los descriptores propuestos para el cultivo de pimiento (Capsicum spp.) (IPGRI, 1995), se seleccionaron las siguientes variables por evaluar:

1. Altura de la planta $(\mathrm{m})$ : se midió la altura de las plantas al final del ciclo del cultivo, a los 209 días después de trasplante (ddt). La altura se midió desde la base del tallo de la planta, hasta el último meristemo de crecimiento apical, con la ayuda de una cinta métrica marca Assist, modelo 32G-8025, con una capacidad de 800,0 \pm $0,1 \mathrm{~cm}$.

2. Área de la hoja (cm2): a los $182 \mathrm{ddt}$ se tomaron ocho hojas ubicadas en la parte central de las plantas, para cada unidad experimental.

Tabla 2

Parámetros de calidad para pimiento cónico

\begin{tabular}{llll}
\hline \multirow{2}{*}{ Parámetro } & \multicolumn{3}{l}{ Categoría de calidad } \\
\cline { 2 - 4 } Longitud del fruto & Primera & Segunda & Rechazo \\
Forma del fruto & Mayor a $12 \mathrm{~cm}$ & Menor a $12 \mathrm{~cm}$ & Menor a $6 \mathrm{~cm}$ \\
$\begin{array}{l}\text { Presencia de manchas, } \\
\text { cicatrices, o quema de sol }\end{array}$ & Sin curvaturas & Con curvaturas & Frutos deformes \\
Estrías o grietas & Ninguna & No mayores a $1 \mathrm{~cm}^{2}$ & Mayores a $1 \mathrm{~cm}^{2}$ \\
& & $\begin{array}{l}\text { No mayores a } 3 \mathrm{~cm} \\
\text { de longitud }\end{array}$ & $\begin{array}{l}\text { Mayores a } 3 \mathrm{~cm} \\
\text { de longitud }\end{array}$ \\
\hline
\end{tabular}


Tabla 3

Parámetros de calidad para pimiento cuadrado y rectangular

\begin{tabular}{llll}
\hline \multirow{2}{*}{ Parámetro } & \multicolumn{3}{l}{ Categoría de calidad } \\
\cline { 2 - 4 } Longitud del fruto & Primera & Segunda & Rechazo \\
Forma del fruto & Puntas bien definidas & Puntas deformes & Frutos muy deformes \\
$\begin{array}{l}\text { Presencia de manchas, } \\
\text { cicatrices, o quema de sol }\end{array}$ & Ninguna & No mayores a $1 \mathrm{~cm}^{2}$ & Mayores a $1 \mathrm{~cm}^{2}$ \\
Estrías o grietas & Ninguna & $\begin{array}{l}\text { No mayores a } 3 \mathrm{~cm} \\
\text { de longitud }\end{array}$ & $\begin{array}{l}\text { Mayores a 3 cm de } \\
\text { longitud }\end{array}$ \\
\hline
\end{tabular}

Cada muestra se evaluó utilizando un medidor de área foliar Modelo Li-3100C, y se calculó el promedio por hoja.

3. Diámetro del tallo $(\mathrm{mm})$ : se realizó una medición a cada una de las plantas de la unidad experimental a los $182 \mathrm{ddt}$; esto se realizó con la ayuda de un calibrador digital marca Mitutoyo, modelo CD, con una capacidad de 15,00 $\pm 0,01$ $\mathrm{cm}$. Esta medición se hizo en la parte media del tallo, antes de la primera bifurcación.

4. Longitud del tallo $(\mathrm{cm})$ : se realizó la medición a los $48 \mathrm{ddt}$, a cada planta de la unidad experimental, y se obtuvo el promedio. La medición se realizó con una cinta métrica, desde la base del tallo de la planta hasta la zona en que inicia la bifurcación del tallo.

5. Ancho del fruto $(\mathrm{cm})$ : se evaluó esta característica a 20 frutos de cada repetición, en frutos que no presentaban deformidades. A cada fruto se le midió sus dimensiones en la sección ecuatorial, con un calibrador digital.

6. Longitud del fruto $(\mathrm{cm})$ : se evaluó esta característica a 20 frutos de cada repetición, en frutos que no presentaba deformidades. A cada fruto se le midió su dimensión longitudinal, con un calibrador digital.
7. Espesor de la pared del fruto (mm): se recolectaron 20 frutos por cada repetición a lo largo de la cosecha. Se realizó un corte transversal en la parte media del fruto, y con un calibrador digital se midió el grosor de la pared del fruto en la parte más ancha.

8. Edad al inicio de la cosecha (ddt): se registró la edad de la planta, desde la fecha del trasplante hasta la fecha en que se cosechó el primer fruto.

9. Número de frutos por planta: se determinó el número total de frutos producidos por planta, así como el número de frutos por cada categoría de calidad; se realizó una sumatoria de los frutos obtenidos en las 20 cosechas de cada repetición, y luego se promedió entre las cuatro plantas de la unidad experimental. Para efectos del presente análisis, se contabilizó el número de frutos de primera calidad, y el número total de frutos, por planta.

10. Peso del fruto de primera calidad (g): se determinó realizando una sumatoria del peso de todos los frutos de primera calidad registrados durante las 20 cosechas de cada repetición, y se dividió entre el número de frutos totales evaluados. Para la evaluación de esta variable, se utilizó una balanza electrónica 
marca Ocony, modelo TH-I-EK, con una capacidad de 5000,0 $\pm 0,1 \mathrm{~g}$.

11. Rendimiento comercial (ton/ha): se calculó a partir del peso total de la cosecha de cada repetición, el cual se dividió entre cuatro plantas para obtener la producción en $\mathrm{kg} /$ planta, y luego se multiplicó por la densidad de siembra, con lo cual se obtuvo el estimado de rendimiento por hectárea; esto se determinó para cada categoría de calidad del fruto. El rendimiento comercial se calculó sumando únicamente el rendimiento de las categorías de primera y segunda calidad.

Se utilizó un diseño experimental irrestricto al azar, donde los tratamientos fueron cada tipo de pimiento; y cada genotipo correspondió a una repetición. La unidad experimental estuvo constituida por dos sacos con cuatro plantas cada una, y la parcela útil se formó por las cuatro plantas ubicadas en la posición central de la unidad experimental. Para las doce variables evaluadas, se obtuvo el coeficiente de correlación de Pearson ( $r$ ) entre ellas, y para aquellas combinaciones de variables en que se obtuvo una correlación alta $(r \geq 0,69)$ y significancia estadística $(p \leq 0,05)$, se obtuvo la regresión lineal con su respectiva ecuación y coeficiente de determinación (R2). Las correlaciones se obtuvieron tanto entre el total de los 27 genotipos evaluados, como según el tipo de pimiento (excepto para el tipo rectangular, en cuyo caso el número de repeticiones fue muy bajo).

\section{Resultados y discusión}

En las tablas 4 al 24, se presentan las correlaciones de Pearson para cada una de las combinaciones de las variables evaluadas, y en las figuras 1 a 21 se presentan las regresiones lineales y sus coeficientes de determinación. Solamente no se presentan los resultados para las combinaciones de variables en que la correlación entre el total de 27 genotipos, o entre los 13 genotipos de pimiento cuadrado, o entre los 12 genotipos de pimiento cónico, resultó ser no significativa.

Con respecto a la correlación entre el área de la hoja y la altura de la planta, el coeficiente obtenido fue alto, positivo, y altamente significativo, tanto para el total de los genotipos evaluados $(r=0,90 ; R 2=0,80)$ (figura 1$)$, como para el grupo de pimiento cónico $(r=0,77 ; R 2$ $=0,59)$ y el de pimiento cuadrado $(r=0,72 ; R 2$ $=0,52$ ) (tabla 4). Unos autores encontraron una correlación positiva y significativa, pero baja, entre ambas variables $(r=0,36)$ (Misra, Lal, Darokar, \& Khanuja, 2010), mientras que otros investigadores hallaron una correlación positiva, muy baja, y no significativa entre ambas variables $(\mathrm{r}=0,08-0,10)$ (Sharma, y otros, 2010; Bijalwan \& Mishra, 2016).

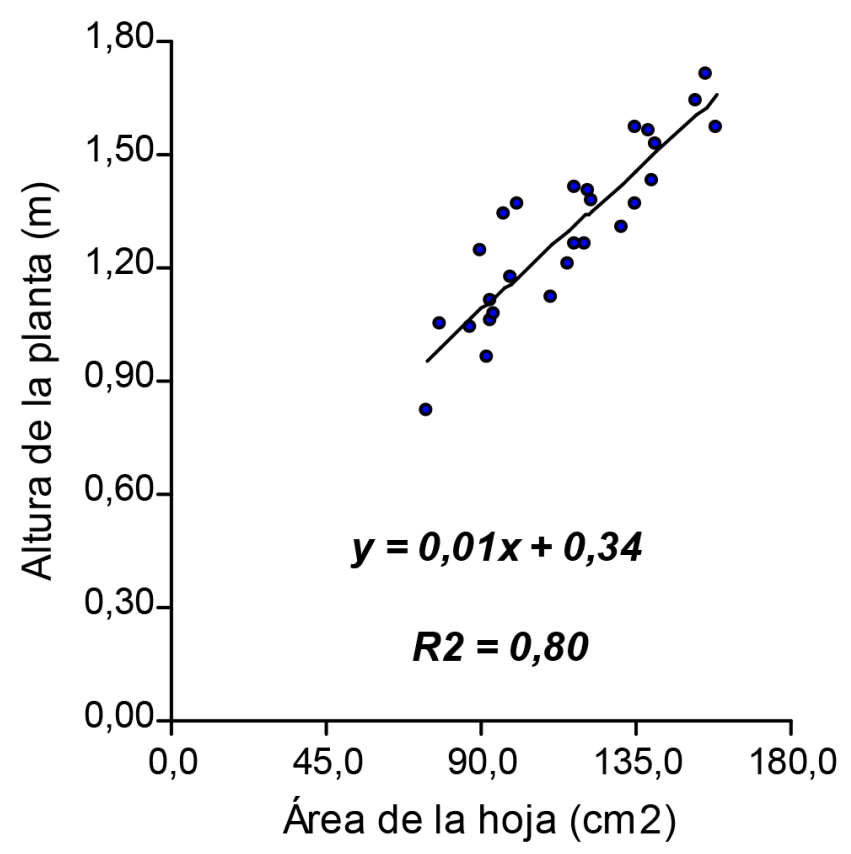

Figura 1. Regresión lineal de la altura de la planta $(\mathrm{m})$ versus el área de la hoja $(\mathrm{cm} 2)$ para el total de genotipos evaluados $(n=27)$. 
Tabla 4

Coeficientes de correlación de Pearson entre altura de la planta y área de la hoja

\begin{tabular}{lccc}
\hline Genotipos & $\begin{array}{c}\text { Coeficiente de } \\
\text { correlación }(\mathrm{r})\end{array}$ & Probabilidad $(\mathrm{p})$ & $\begin{array}{c}\text { Coeficiente de determinación de la } \\
\text { regresión lineal (R2) }\end{array}$ \\
\hline Total $(\mathrm{n}=27)$ & 0,90 & $* *$ & 0,80 \\
Cónico $(\mathrm{n}=12)$ & 0,77 & $* *$ & 0,59 \\
Cuadrado $(\mathrm{n}=13)$ & 0,72 & $* *$ & 0,52 \\
\hline
\end{tabular}

Nota $. \mathrm{ns}=$ no significativo ${ }^{*}=$ significativa $(\mathrm{p} \leq 0,05){ }^{* *}=$ altamente significativa $(\mathrm{p} \leq 0,01)$.

Se halló una correlación alta, negativa, y altamente significativa, entre el ancho del fruto y el área de la hoja para el total de genotipos evaluados $(r=-0,71 ; R 2=0,50)$ (figura 2$)$, pero en el grupo de pimiento cónico o el de pimiento cuadrado dicha correlación fue baja, positiva y no significativa (tabla 5). En forma contraria a lo encontrado en el presente ensayo, en otro estudio se obtuvo una correlación alta, positiva, y altamente significativa entre ambas variables ( $\mathrm{r}=0,71)$ (Sharma, y otros, 2010); otros autores hallaron una correlación positiva y altamente significativa, pero no tan alta entre esas variables $(r=0,46)$ (Misra, y otros, 2010), y en otra investigación la correlación obtenida fue positiva, baja y no significativa $(\mathrm{r}=0,13)$ (Bijalwan \& Mishra, 2016).

Se encontró una correlación alta, positiva, y altamente significativa, entre el número total de frutos por planta y el área de la hoja para el total de genotipos evaluados $(r=0,77 ; R 2=0,59)$ (figura $3)$, mientras que para los grupos de pimiento cónico o cuadrado dicha correlación no fue significativa (tabla 6). En otros estudios, se obtuvo una correlación positiva, baja, y no significativa entre ambas variables $(r=0,03-0,24)$ (Sharma, y otros, 2010; Bijalwan \& Mishra, 2016).

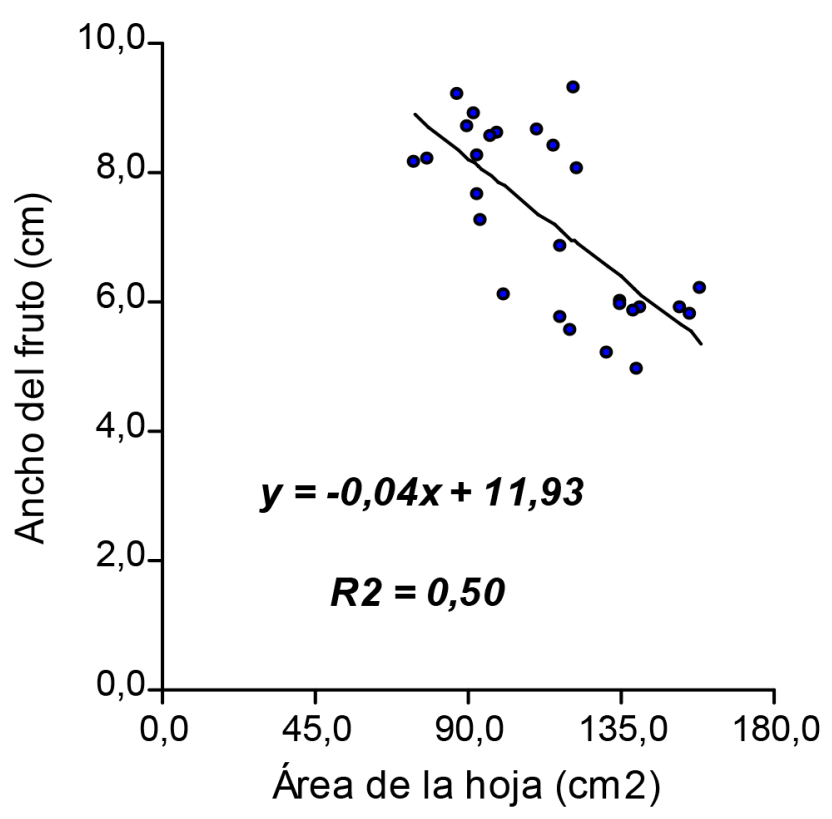

Figura 2. Regresión lineal del ancho del fruto (cm) versus el área de la hoja ( $\mathrm{cm} 2)$ para el total de genotipos evaluados $(n=27)$. 
Elizondo-Cabalcetan y Monge-Pérez

Tabla 5

Coeficientes de correlación de Pearson entre ancho del fruto y área de la hoja

\begin{tabular}{lccc}
\hline Genotipos & $\begin{array}{c}\text { Coeficiente de } \\
\text { correlación }(\mathrm{r})\end{array}$ & Probabilidad $(\mathrm{p})$ & $\begin{array}{c}\text { Coeficiente de determinación de la } \\
\text { regresión lineal (R2) }\end{array}$ \\
\hline Total $(\mathrm{n}=27)$ & $-0,71$ & $* *$ & 0,50 \\
Cónico $(\mathrm{n}=12)$ & 0,06 & $\mathrm{~ns}$ & \\
Cuadrado $(\mathrm{n}=13)$ & 0,33 & $\mathrm{~ns}$ & \\
\hline
\end{tabular}

Nota. $\mathrm{ns}=$ no significativo; ${ }^{*}=$ significativa $(\mathrm{p} \leq 0,05) ;{ }^{*}=$ altamente significativa $(\mathrm{p} \leq 0,01)$.

Tabla 6

Coeficientes de correlación de Pearson entre número total de frutos por planta y área de la hoja

\begin{tabular}{lccc}
\hline Genotipos & $\begin{array}{c}\text { Coeficiente de } \\
\text { correlación }(\mathrm{r})\end{array}$ & Probabilidad $(\mathrm{p})$ & $\begin{array}{c}\text { Coeficiente de determinación de la } \\
\text { regresión lineal (R2) }\end{array}$ \\
\hline Total $(\mathrm{n}=27)$ & 0,77 & $* *$ & 0,59 \\
Cónico $(\mathrm{n}=12)$ & 0,51 & $\mathrm{~ns}$ & \\
Cuadrado $(\mathrm{n}=13)$ & $-0,12$ & $\mathrm{~ns}$ & \\
\hline
\end{tabular}

Nota $. \mathrm{ns}=$ no significativo; ${ }^{*}=$ significativa $(\mathrm{p} \leq 0,05) ;{ }^{* *}=$ altamente significativa $(\mathrm{p} \leq 0,01)$.

Se halló una correlación alta, negativa, y altamente significativa, entre el espesor de la pared del fruto y la altura de la planta para el total de genotipos evaluados $(r=-0,78$; $\mathrm{R} 2=$ 0,61 ) (figura 5), pero la correlación negativa no fue estadísticamente significativa para el grupo de pimiento cónico o el de pimiento cuadrado (tabla 8). Otros investigadores encontraron una correlación negativa, muy baja, y no significativa entre ambas variables $(\mathrm{r}=-0,02 \mathrm{a}-0,16)$ (Sharma, y otros, 2010; Mathew, Doijode, \& Reddy, 2004; Bundela, Pant, Madhuri, \& Singh, 2018); mientras que en otros estudios la correlación hallada también fue muy baja y no significativa, pero positiva ( $\mathrm{r}=0,01-0,11$ ) (Bijalwan \& Mishra, 2016; Aklilu, Abebie, Wogari, \& Wolde, 2016; Shumbulo, Nigussie, \& Alamerew, 2017). Por otra parte, en Turquía se obtuvo una correlación positiva y altamente significativa, aunque baja $(\mathrm{r}=0,21)$ (Cancaya, y otros, 2010). Y en otra investigación, los valores obtenidos fueron ( $\mathrm{r}=$ $-0,41$ a -0,44) (Ben-Chaim \& Paran, 2000).

Se encontró una correlación alta, positiva, y altamente significativa, entre el espesor de la pared del fruto y el ancho del fruto para el total de genotipos evaluados $(r=0,86 ; R 2=0,73$ ) (figura 6), mientras que para los grupos de pimiento cónico o cuadrado dicha correlación fue muy baja y no fue significativa (tabla 9). En otras investigaciones se halló también una correlación alta, positiva y altamente significativa entre esas variables $(r=0,68$ -0,88) (Mathew, y otros, 2004; Silva, Nascimento, Cecon, Sapucay, Rêgo, \& Barbosa, 2013). Unos 


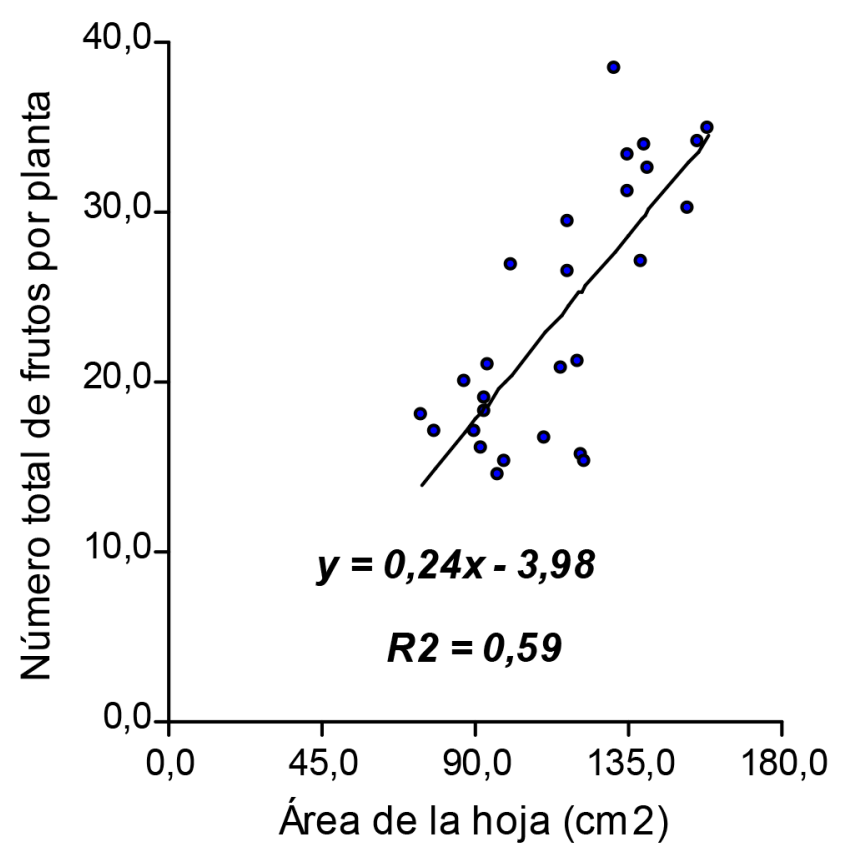

Figura 3. Regresión lineal del número total de frutos por planta versus el área de la hoja $(\mathrm{cm} 2)$ para el total de genotipos evaluados $(n=27)$.

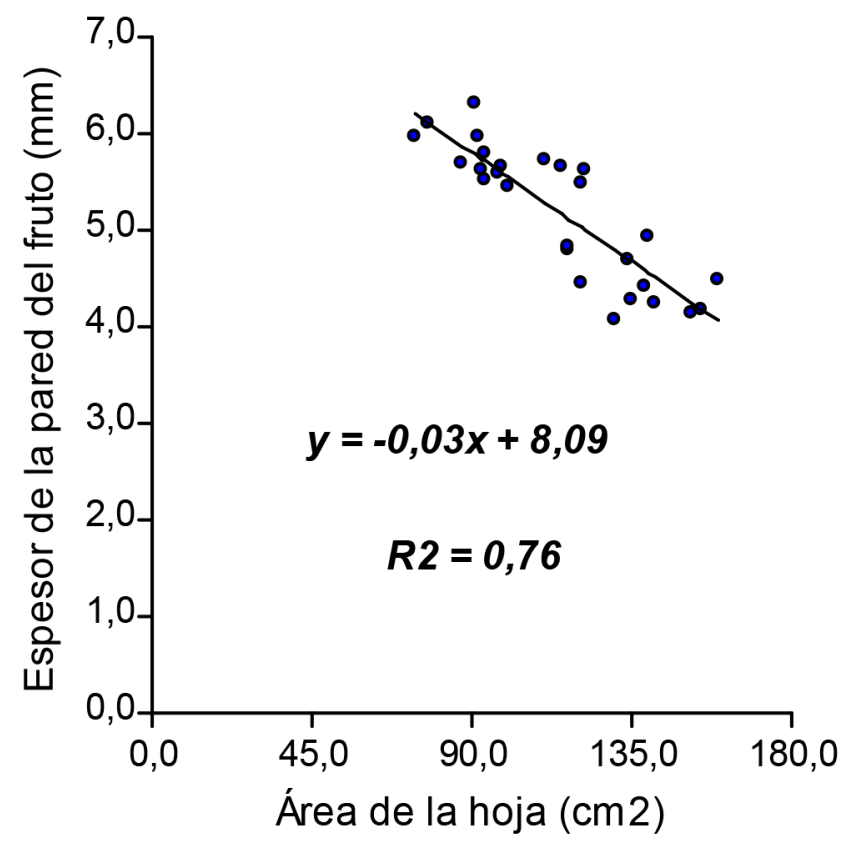

Figura 4. Regresión lineal del espesor de la pared del fruto $(\mathrm{mm})$ versus el área de la hoja $(\mathrm{cm} 2)$ para el total de genotipos evaluados $(n=27)$.

Tabla 7

Coeficientes de correlación de Pearson entre espesor de la pared del fruto y área de la hoja

\begin{tabular}{lccc}
\hline Genotipos & $\begin{array}{c}\text { Coeficiente de } \\
\text { correlación }(\mathrm{r})\end{array}$ & $\begin{array}{c}\text { Probabilidad }(\mathrm{p}) \\
\text { Total }(\mathrm{n}=27)\end{array}$ & $\begin{array}{c}\text { Coeficiente de determinación de la } \\
\text { regresión lineal }(\mathrm{R} 2)\end{array}$ \\
Cónico $(\mathrm{n}=12)$ & $-0,87$ & $* *$ & 0,76 \\
Cuadrado $(\mathrm{n}=13)$ & $-0,66$ & $*$ & \\
\hline
\end{tabular}

Nota. $\mathrm{ns}=$ no significativo; ${ }^{*}=$ significativa $(\mathrm{p} \leq 0,05) ;{ }^{* *}=$ altamente significativa $(\mathrm{p} \leq 0,01)$. 


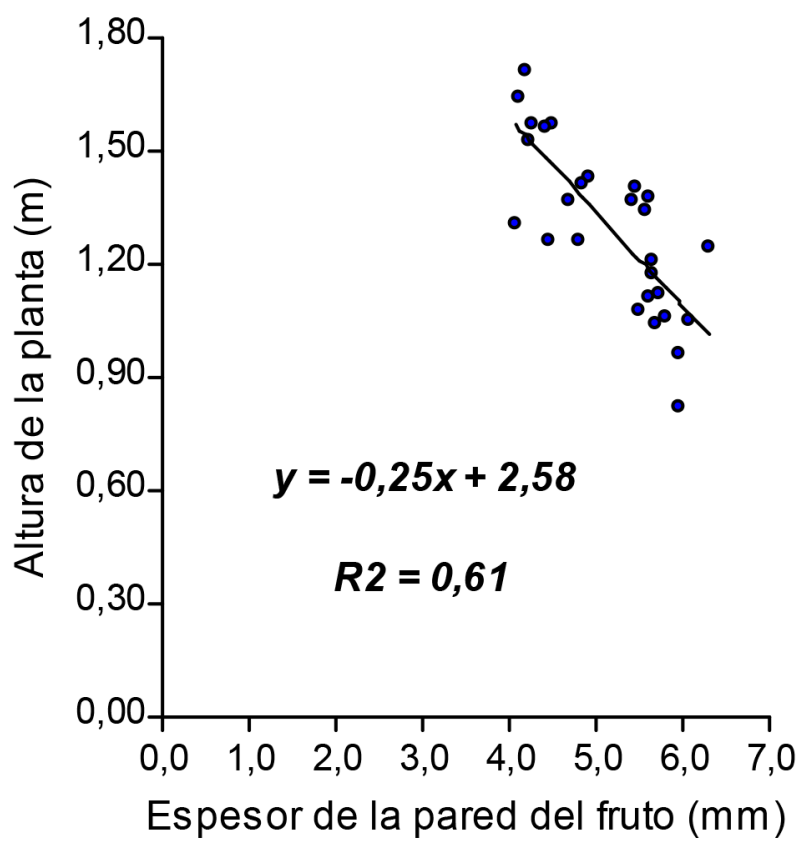

Figura 5. Regresión lineal de la altura de la planta (m) versus el espesor de la pared del fruto $(\mathrm{mm})$ para el total de genotipos evaluados $(n=27)$.

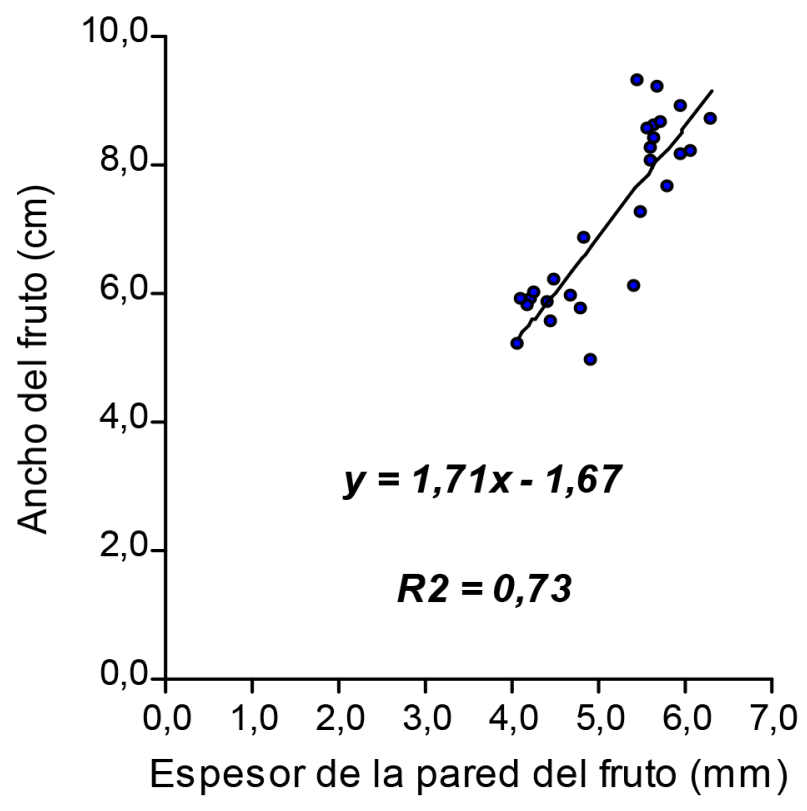

Figura 6. Regresión lineal del ancho del fruto $(\mathrm{cm})$ versus el espesor de la pared del fruto $(\mathrm{mm})$ para el total de genotipos evaluados $(n=27)$.

Tabla 8

Coeficientes de correlación de Pearson entre espesor de la pared del fruto y altura de la planta

\begin{tabular}{lccc}
\hline Genotipos & $\begin{array}{c}\text { Coeficiente de } \\
\text { correlación }(\mathrm{r})\end{array}$ & Probabilidad $(\mathrm{p})$ & $\begin{array}{c}\text { Coeficiente de determinación de la } \\
\text { regresión lineal }(\mathrm{R} 2)\end{array}$ \\
\hline Total $(\mathrm{n}=27)$ & $-0,78$ & $* *$ & 0,61 \\
Cónico $(\mathrm{n}=12)$ & $-0,45$ & $\mathrm{~ns}$ & \\
Cuadrado $(\mathrm{n}=13)$ & $-0,39$ & $\mathrm{~ns}$ & \\
\hline
\end{tabular}

Nota. $\mathrm{ns}=$ no significativo; ${ }^{*}=$ significativa $(\mathrm{p} \leq 0,05){ }^{* *}=$ altamente significativa $(\mathrm{p} \leq 0,01)$. 
Pimiento (Capsicum annuum) cultivado bajo invernadero

Tabla 9

Coeficientes de correlación de Pearson entre espesor de la pared del fruto y ancho del fruto

\begin{tabular}{lccc}
\hline Genotipos & $\begin{array}{c}\text { Coeficiente de } \\
\text { correlación }(\mathrm{r})\end{array}$ & Probabilidad $(\mathrm{p})$ & $\begin{array}{c}\text { Coeficiente de determinación de la } \\
\text { regresión lineal (R2) }\end{array}$ \\
\hline Total $(\mathrm{n}=27)$ & 0,86 & $* *$ & 0,73 \\
Cónico $(\mathrm{n}=12)$ & 0,08 & $\mathrm{~ns}$ & \\
Cuadrado $(\mathrm{n}=13)$ & 0,03 & $\mathrm{~ns}$ & \\
\hline
\end{tabular}

Nota. $\mathrm{ns}=$ no significativo ${ }^{*}=$ significativa $(\mathrm{p} \leq 0,05) ;{ }^{* *}=$ altamente significativa $(\mathrm{p} \leq 0,01)$.

autores obtuvieron una correlación positiva, no muy alta, y no significativa entre ambas variables $(\mathrm{r}=0,30-0,65)$ (Sharma, y otros, 2010; Aklilu, y otros, 2016; Shumbulo, y otros, 2017). Por otra parte, en otros estudios se halló una correlación positiva, y significativa o altamente significativa, pero no muy alta $(\mathrm{r}=0,32-0,47)$ (Madosa, y otros, 2008; Bijalwan \& Mishra, 2016; Bundela, y otros, 2018; Cancaya, y otros, 2010). Y en otro estudio, los valores obtenidos fueron $(r=0,85-0,86)$ (BenChaim \& Paran, 2000).

Se obtuvo una correlación alta, negativa, y altamente significativa, entre el espesor de la pared del fruto y la longitud del fruto para el total de genotipos evaluados $(r=-0,73$; $R 2=$ 0,53 ) (figura 7 ), mientras que para los grupos de pimiento cónico o cuadrado dicha correlación fue baja y no fue significativa (tabla 10). En otros estudios, se encontró una correlación negativa, muy baja, y no significativa entre ambas variables $(\mathrm{r}=-0,04$ a $-0,16)$ (Sharma, $\mathrm{y}$ otros, 2010; Cancaya, y otros, 2010; Madosa, y otros, 2008; Ben-Chaim \& Paran, 2000; Bijalwan \& Mishra, 2016). Otros autores hallaron una correlación positiva, muy baja, y no significativa entre esas variables $(r=0,04-0,08)$ (Mathew, $y$ otros, 2004; Silva, y otros, 2013). Sin embargo, en otras investigaciones se obtuvo una correlación positiva y altamente significativa, aunque no muy alta entre ambas variables $(r=0,39-0,60)$ (Bundela, y otros, 2018; Aklilu, y otros, 2016; Shumbulo, y otros, 2017).

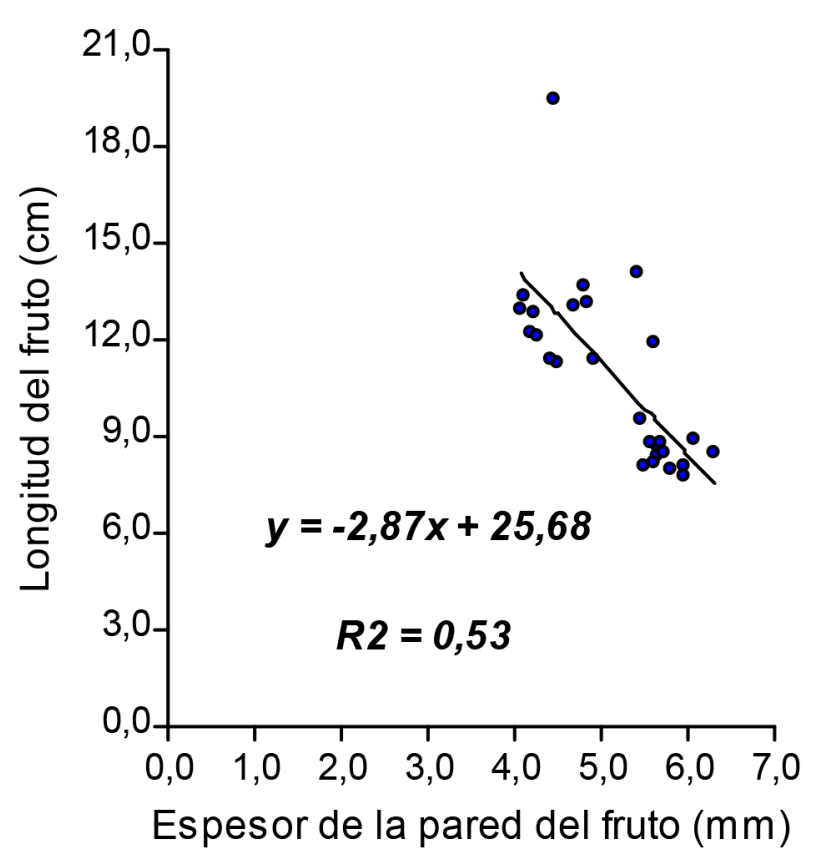

Figura 7. Regresión lineal de la longitud del fruto $(\mathrm{cm})$ versus el espesor de la pared del fruto $(\mathrm{mm})$ para el total de genotipos evaluados $(n=27)$. 
Elizondo-Cabalcetan y Monge-Pérez

Tabla 10

Coeficientes de correlación de Pearson entre espesor de la pared del fruto y longitud del fruto

\begin{tabular}{lccc}
\hline Genotipos & $\begin{array}{c}\text { Coeficiente de } \\
\text { correlación }(\mathrm{r})\end{array}$ & Probabilidad $(\mathrm{p})$ & $\begin{array}{c}\text { Coeficiente de determinación de la } \\
\text { regresión lineal (R2) }\end{array}$ \\
\hline Total $(\mathrm{n}=27)$ & $-0,73$ & $* *$ & 0,53 \\
Cónico $(\mathrm{n}=12)$ & 0,09 & $\mathrm{~ns}$ & \\
Cuadrado $(\mathrm{n}=13)$ & $-0,28$ & $\mathrm{~ns}$ & \\
\hline
\end{tabular}

Nota. $\mathrm{ns}=$ no significativo; ${ }^{*}=$ significativa $(\mathrm{p} \leq 0,05) ;{ }^{* *}=$ altamente significativa $(\mathrm{p} \leq 0,01)$.

En relación al peso del fruto de primera calidad y el espesor de la pared del fruto, se encontró una correlación alta, positiva, y altamente significativa para el total de genotipos evaluados $(\mathrm{r}=0,76 ; \mathrm{R} 2=0,58)$ (figura 8), mientras que para los grupos de pimiento cónico o cuadrado dicha correlación fue muy baja y no significativa (tabla 11). En otras investigaciones también se obtuvo una correlación alta, positiva, y altamente significativa, entre estas variables $(r=0,78-$ 0,88) (Mathew, y otros, 2004; Bundela, y otros, 2018; Silva, y otros, 2013). Otros investigadores hallaron una correlación positiva, muy baja, y no significativa entre ambas variables $(\mathrm{r}=0,12$ 0,19) (Sharma, y otros, 2010; Bijalwan \& Mishra, 2016). Por otra parte, en otros estudios sí se obtuvo una correlación positiva y altamente significativa, aunque no muy alta $(\mathrm{r}=0,23-0,54)$ (Madosa, y otros, 2008; Cancaya, y otros, 2010; Aklilu, y otros, 2016). Y en otra investigación, los valores obtenidos fueron $(r=0,63-0,85)$ (Ben-Chaim \& Paran, 2000).

Con respecto al número total de frutos por planta y el espesor de la pared del fruto, se halló una correlación alta, negativa, y altamente significativa para el total de genotipos evaluados $(\mathrm{r}=-0,85 ; \mathrm{R} 2=0,73)$ (figura 9), mientras que para los grupos de pimiento cónico o cuadrado dicha correlación fue negativa, baja y no significativa (tabla 12). En otra investigación, también se halló una correlación negativa, alta, y altamente significativa entre estas variables $(\mathrm{r}=-0,73)$ (Bundela, y otros, 2018). Otros autores obtuvieron una correlación negativa,

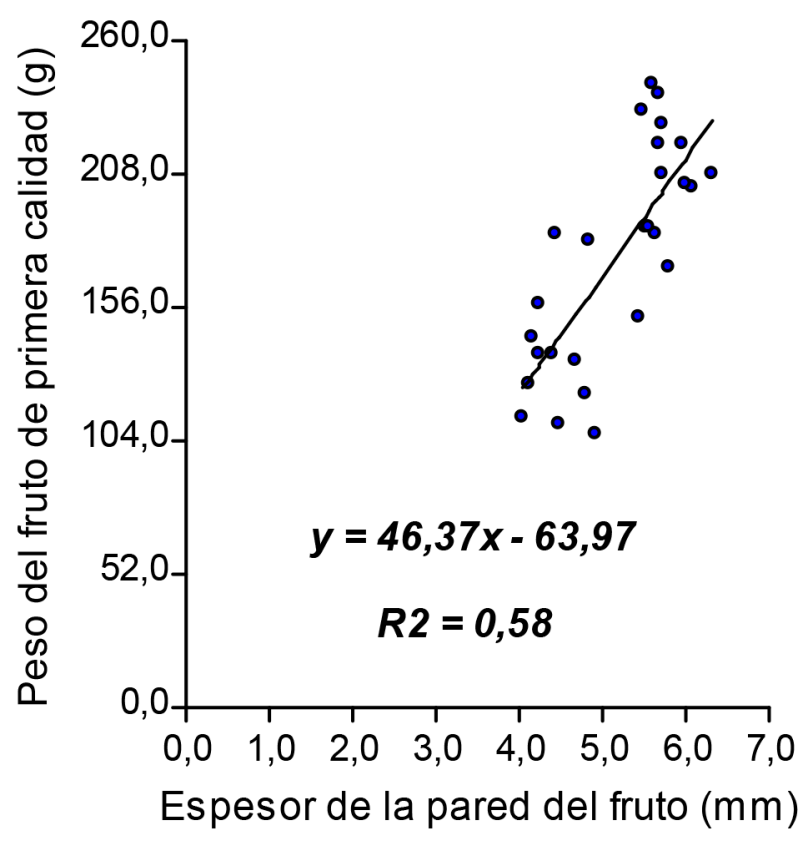

Figura 8. Regresión lineal del peso del fruto de primera calidad (g) versus el espesor de la pared del fruto $(\mathrm{mm})$ para el total de genotipos evaluados $(n=27)$. 
Pimiento (Capsicum annuum) cultivado bajo invernadero

Tabla 11

Coeficientes de correlación de Pearson entre peso del fruto de primera calidad y espesor de la pared del fruto

\begin{tabular}{lccc}
\hline Genotipos & $\begin{array}{c}\text { Coeficiente de } \\
\text { correlación }(\mathrm{r})\end{array}$ & Probabilidad $(\mathrm{p})$ & $\begin{array}{c}\text { Coeficiente de determinación de la } \\
\text { regresión lineal }(\mathrm{R} 2)\end{array}$ \\
\hline Total $(\mathrm{n}=27)$ & 0,76 & $* *$ & 0,58 \\
Cónico $(\mathrm{n}=12)$ & 0,01 & ns & \\
Cuadrado $(\mathrm{n}=13)$ & $-0,02$ & ns
\end{tabular}

Nota. $\mathrm{ns}=$ no significativo; ${ }^{*}=$ significativa $(\mathrm{p} \leq 0,05) ;{ }^{* *}=$ altamente significativa $(\mathrm{p} \leq 0,01)$.

Tabla 12

Coeficientes de correlación de Pearson entre número total de frutos por planta y espesor de la pared del fruto

\begin{tabular}{lccc}
\hline Genotipos & $\begin{array}{c}\text { Coeficiente de } \\
\text { correlación }(\mathrm{r})\end{array}$ & Probabilidad $(\mathrm{p})$ & $\begin{array}{c}\text { Coeficiente de determinación de la } \\
\text { regresión lineal }(\mathrm{R} 2)\end{array}$ \\
\hline Total $(\mathrm{n}=27)$ & $-0,85$ & $* *$ & 0,73 \\
Cónico $(\mathrm{n}=12)$ & $-0,32$ & ns & \\
Cuadrado $(\mathrm{n}=13)$ & $-0,09$ & $\mathrm{~ns}$
\end{tabular}

Nota. $\mathrm{ns}=$ no significativo; ${ }^{*}=$ significativa $(\mathrm{p} \leq 0,05) ;{ }^{* *}=$ altamente significativa $(\mathrm{p} \leq 0,01)$.

significativa, pero baja, entre ambas variables ( $\mathrm{r}$ $=-0,31$ ) (Mathew, y otros, 2004). Por otra parte, en otros estudios se encontró una correlación negativa, baja, y no significativa entre esas variables ( $\mathrm{r}=-0,02$ a $-0,31$ ) (Madosa, y otros, 2008; Bijalwan \& Mishra, 2016; Aklilu, y otros, 2016; Shumbulo, y otros, 2017). En Turquía se obtuvo una correlación positiva y altamente significativa, pero baja $(\mathrm{r}=0,28)$ (Cancaya, $\mathrm{y}$ otros, 2010). En India se obtuvo una correlación positiva, baja, y no significativa entre ambas variables $(r=0,18)$ (Sharma, y otros, 2010).

En relación al número total de frutos por planta y el ancho del fruto, se encontró una correlación alta, negativa, y altamente significativa, para el total de genotipos evaluados $(r=-0,89 ; R 2=0,79)$ (figura 10), mientras que para los grupos de pimiento cónico o cuadrado dicha correlación fue baja y no fue significativa (tabla 13). En otras investigaciones se obtuvo una correlación negativa, significativa o altamente significativa, pero no muy alta, entre estas variables $(\mathrm{r}=$ -0,21 a -0,62) (Tembhurne \& Rao, 2013; Vijaya, Gowda, \& Nehru, 2014; Yatung, Dubey, Singh, Upadhyay, \& Pandey, 2014; Chakrabarty \& Islam, 2017; Shweta, Basavarajappa, Satish, Jagadeesha, Hanachinmani, \& Dilepkumar, 2018). Por otra parte, en otros estudios se halló una correlación negativa, baja, y no significativa ( $\mathrm{r}=-0,11$ a -0,27) (Sasu, y otros, 2013; Madosa, y otros, 2008; Pandit \& Adhikary, 2014; Vikram, Warshamana, \& Gupta, 2014; Aklilu, 


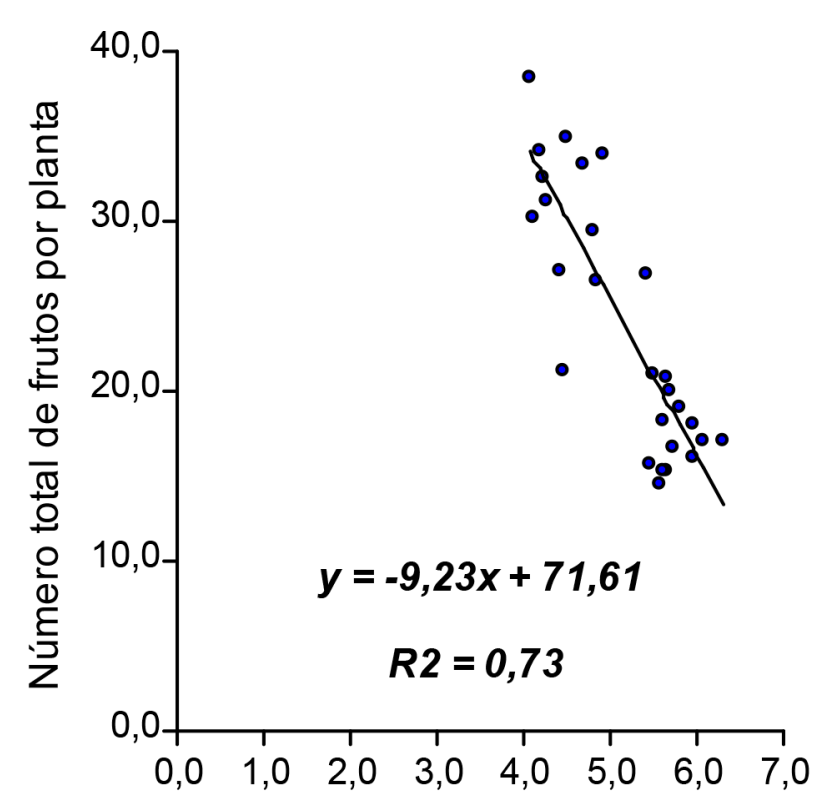

Espesor de la pared del fruto $(\mathrm{mm})$

Figura 9. Regresión lineal del número total de frutos por planta versus el espesor de la pared del fruto $(\mathrm{mm})$ para el total de genotipos evaluados $(\mathrm{n}=27)$.

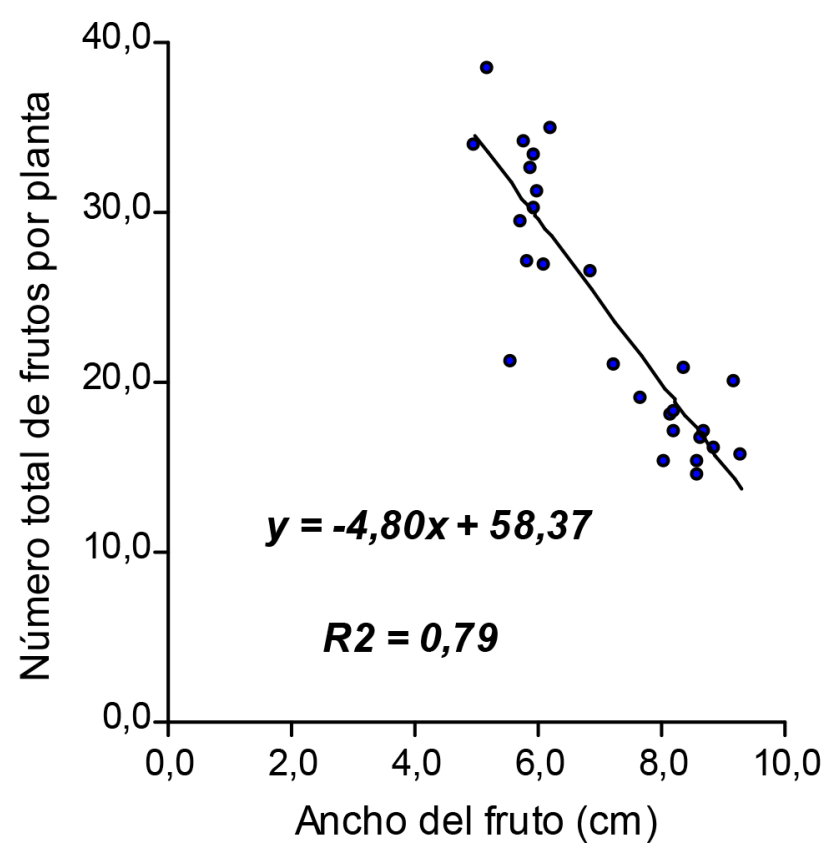

Figura 10. Regresión lineal del número total de frutos por planta versus el ancho del fruto $(\mathrm{cm})$ para el total de genotipos evaluados $(\mathrm{n}=27)$.

Tabla 13

Coeficientes de correlación de Pearson entre número total de frutos por planta y ancho del fruto

\begin{tabular}{lccc}
\hline Genotipos & $\begin{array}{c}\text { Coeficiente de } \\
\text { correlación }(\mathrm{r})\end{array}$ & Probabilidad $(\mathrm{p})$ & $\begin{array}{c}\text { Coeficiente de determinación de la } \\
\text { regresión lineal (R2) }\end{array}$ \\
\hline Total $(\mathrm{n}=27)$ & $-0,89$ & $* *$ & 0,79 \\
Cónico $(\mathrm{n}=12)$ & $-0,23$ & $\mathrm{~ns}$ & \\
Cuadrado $(\mathrm{n}=13)$ & $-0,51$ & $\mathrm{~ns}$ & \\
\hline
\end{tabular}

Nota. $\mathrm{ns}=$ no significativo; ${ }^{*}=$ significativa $(\mathrm{p} \leq 0,05) ;{ }^{* *}=$ altamente significativa $(\mathrm{p} \leq 0,01)$. 
y otros, 2016; Rokib, Matin, Nazmul, Abul, \& Mahmudul, 2016; Shumbulo, y otros, 2017). Otros autores encontraron una correlación positiva, significativa o altamente significativa, aunque no muy alta $(\mathrm{r}=0,17-0,65)$ (Cancaya, y otros, 2010; Mathew, y otros, 2004; Bundela, y otros, 2018; Pujar, Tirakannanavar, Jagadeesha, Gasti, \& Sandhyarani, 2017). Y otros investigadores obtuvieron una correlación positiva, baja, y no significativa entre ambas variables $(\mathrm{r}=0,10-0,31)$ (Sharma, y otros, 2010; Bijalwan \& Mishra, 2016).

Se obtuvo una correlación alta, negativa, y altamente significativa, entre el ancho del fruto y la longitud del tallo para el total de genotipos evaluados $(r=-0,73 ; \mathrm{R} 2=0,53)$ (figura $11)$, mientras que para los grupos de pimiento cónico o cuadrado dicha correlación negativa fue baja, y no fue significativa (tabla 14).

Con respecto a la relación entre longitud del fruto y ancho del fruto, se encontró una correlación alta, negativa, y altamente significativa para el total de genotipos evaluados $(\mathrm{r}=-0,76 ; \mathrm{R} 2=0,58)$ (figura 12), mientras que para el grupo de pimiento cónico dicha correlación fue negativa, baja, y no fue significativa $(r=$ $-0,08)$, y para el grupo de pimiento cuadrado fue positiva y significativa, pero no fue alta $(\mathrm{r}$

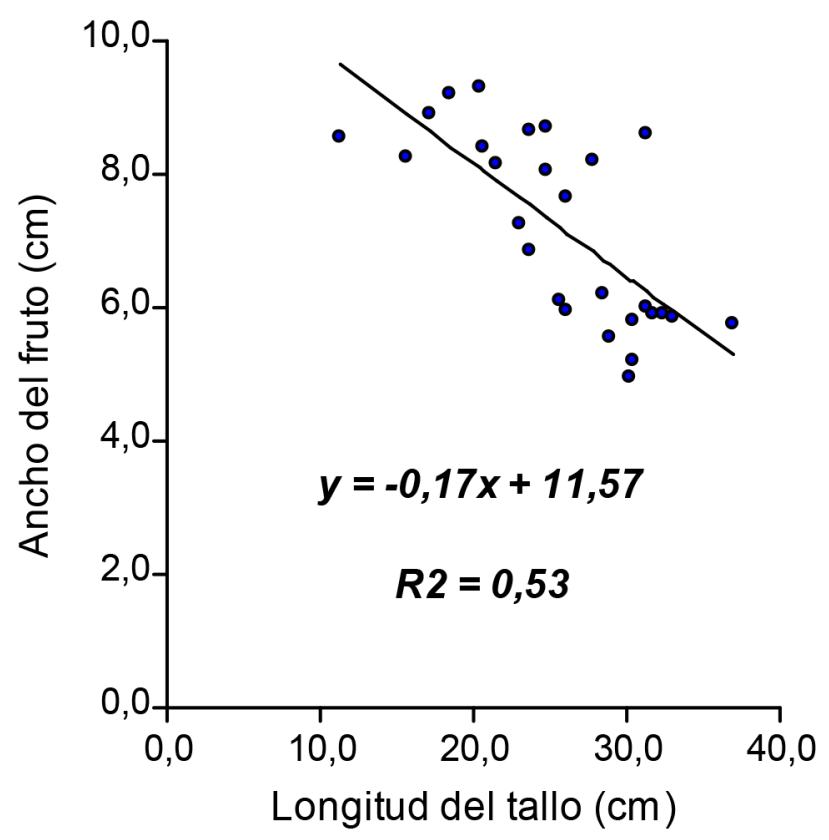

Figura 11. Regresión lineal del ancho del fruto (cm) versus la longitud del tallo $(\mathrm{cm})$ para el total de genotipos evaluados $(n=27)$.

\section{Tabla 14}

Coeficientes de correlación de Pearson entre ancho del fruto y longitud del tallo

\begin{tabular}{lccc}
\hline Genotipos & $\begin{array}{c}\text { Coeficiente de } \\
\text { correlación }(\mathrm{r})\end{array}$ & Probabilidad $(\mathrm{p})$ & $\begin{array}{c}\text { Coeficiente de determinación de la } \\
\text { regresión lineal (R2) }\end{array}$ \\
\hline Total $(\mathrm{n}=27)$ & $-0,73$ & $* *$ & 0,53 \\
Cónico $(\mathrm{n}=12)$ & $-0,18$ & $\mathrm{~ns}$ & \\
Cuadrado $(\mathrm{n}=13)$ & $-0,25$ & $\mathrm{~ns}$ & \\
\hline
\end{tabular}

Nota. $\mathrm{ns}=$ no significativo; ${ }^{*}=$ significativa $(\mathrm{p} \leq 0,05) ;{ }^{* *}=$ altamente significativa $(\mathrm{p} \leq 0,01)$. 
$=0,63$ ) (tabla 15). En otras investigaciones se obtuvo una correlación negativa y significativa, pero baja, entre estas variables $(r=-0,32$ a $-0,35)$ (Pandit \& Adhikary, 2014; Vijaya, y otros, 2014; Rokib, y otros, 2016). De manera contraria a lo obtenido en la presente investigación, otros investigadores hallaron una correlación alta, positiva, y altamente significativa entre ambas variables $(\mathrm{r}=0,80-0,90)$ (Sharma, $\mathrm{y}$ otros, 2010; Chakrabarty \& Islam, 2017). Otros autores encontraron una correlación positiva y significativa, pero baja, entre esas variables ( $\mathrm{r}$ $=0,18-0,31$ ) (Pujar, y otros, 2017; Shumbulo, y otros, 2017; Jogi, Madalageri, Ganiger, Bhuvaneswari, Patil, \& Kotikal, 2013). En otros estudios se obtuvo una correlación positiva, baja, y no significativa entre estas características $(r=0,01-0,30)$ (Sasu, y otros, 2013; Cancaya, y

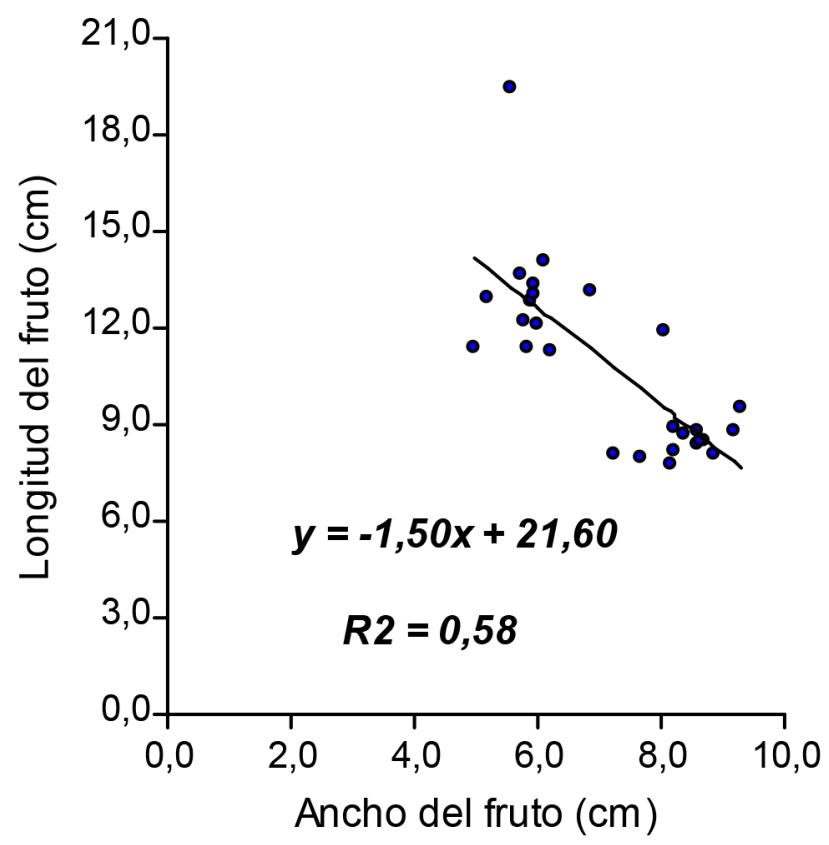

Figura 12. Regresión lineal de la longitud del fruto $(\mathrm{cm})$ versus el ancho del fruto $(\mathrm{cm})$ para el total de genotipos evaluados $(n=27)$. otros, 2010; Bijalwan \& Mishra, 2016; Vikram, y otros, 2014; Tembhurne \& Rao, 2013; Misra, y otros, 2010; Bundela, y otros, 2018; Aklilu, y otros, 2016; Silva, y otros, 2013; Shweta, y otros, 2018). Y en otras investigaciones se halló una correlación muy baja, negativa, y no significativa $(\mathrm{r}=-0,02 \mathrm{a}-0,12)$ (Madosa, y otros, 2008; Ben-Chaim \& Paran, 2000; Mathew, y otros, 2004; Yatung, y otros, 2014).

Se halló una correlación alta, positiva, y altamente significativa, entre el peso del fruto de primera calidad y el ancho del fruto para el total de genotipos evaluados $(r=0,89 ; R 2=0,79)$ (figura 13), al igual que para el grupo de pimiento cuadrado $(r=0,71 ; R 2=0,50)$, mientras que para el grupo de pimiento cónico dicha correlación fue baja y no fue significativa (tabla 16). En otros

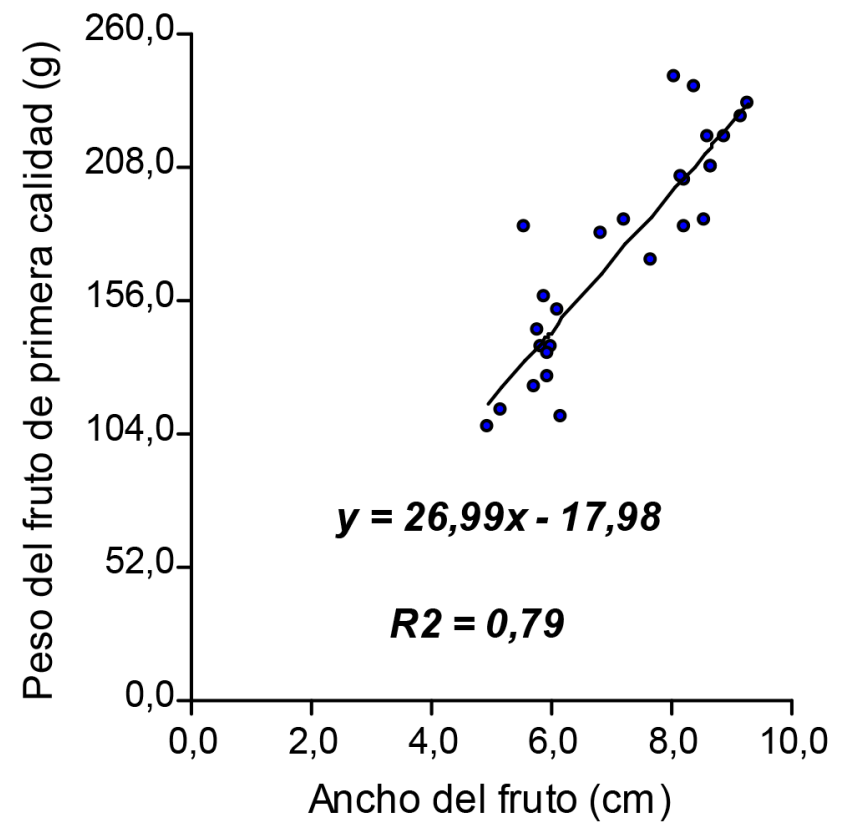

Figura 13. Regresión lineal del peso del fruto de primera calidad (g) versus el ancho del fruto $(\mathrm{cm})$ para el total de genotipos evaluados $(n=27)$. 
Pimiento (Capsicum annuum) cultivado bajo invernadero

Tabla 15

Coeficientes de correlación de Pearson entre longitud del fruto y ancho del fruto

\begin{tabular}{lccc}
\hline Genotipos & $\begin{array}{c}\text { Coeficiente de } \\
\text { correlación }(\mathrm{r})\end{array}$ & Probabilidad $(\mathrm{p})$ & $\begin{array}{c}\text { Coeficiente de determinación de la } \\
\text { regresión lineal (R2) }\end{array}$ \\
\hline Total $(\mathrm{n}=27)$ & $-0,76$ & $* *$ & 0,58 \\
Cónico $(\mathrm{n}=12)$ & $-0,08$ & $\mathrm{~ns}$ & \\
Cuadrado $(\mathrm{n}=13)$ & 0,63 & $*$ & \\
\hline
\end{tabular}

Nota. Elizondo y Monge, $2019 ; \mathrm{ns}=$ no significativo $;{ }^{*}=$ significativa $(\mathrm{p} \leq 0,05) ;{ }^{* *}=$ altamente significativa $(\mathrm{p} \leq 0,01)$.

Tabla 16

Coeficientes de correlación de Pearson entre peso del fruto de primera calidad y ancho del fruto

\begin{tabular}{lccc}
\hline Genotipos & $\begin{array}{c}\text { Coeficiente de } \\
\text { correlación }(\mathrm{r})\end{array}$ & Probabilidad $(\mathrm{p})$ & $\begin{array}{c}\text { Coeficiente de determinación de la } \\
\text { regresión lineal (R2) }\end{array}$ \\
\hline Total $(\mathrm{n}=27)$ & 0,89 & $* *$ & 0.79 \\
Cónico $(\mathrm{n}=12)$ & 0,27 & $\mathrm{~ns}$ & 0,50 \\
Cuadrado $(\mathrm{n}=13)$ & 0,71 & $* *$ & 0 \\
\hline
\end{tabular}

Nota. $\mathrm{ns}=$ no significativo; ${ }^{*}=$ significativa $(\mathrm{p} \leq 0,05) ;{ }^{* *}=$ altamente significativa $(\mathrm{p} \leq 0,01)$.

estudios se encontró también una correlación alta, positiva y altamente significativa entre ambas variables $(\mathrm{r}=0,76-0,93)$ (Madosa, y otros, 2008; Misra, y otros, 2010; Mathew, y otros, 2004; Yatung, y otros, 2014; Chakrabarty \& Islam, 2017; Silva, y otros, 2013). Otros autores hallaron correlaciones positivas y altamente significativas, pero no muy altas $(\mathrm{r}=0,28-0,63)$ (Sharma, $\mathrm{y}$ otros, 2010; Cancaya, y otros, 2010; Sasu, y otros, 2013; Tembhurne \& Rao, 2013; Bundela, y otros, 2018; Pujar, y otros, 2017; Rokib, y otros, 2016). Otros investigadores obtuvieron valores entre $(\mathrm{r}=0,67-0,89)$ (Ben-Chaim \& Paran, 2000). En otros casos, se obtuvieron correlaciones positivas, bajas y no significativas $(\mathrm{r}=0,12-0,37)$ (Pandit \& Adhikary, 2014; Bijalwan \& Mishra,
2016; Vikram, y otros, 2014; Aklilu, y otros, 2016; Shweta, y otros, 2018). Y solamente en un caso se halló una correlación negativa, significativa, pero baja $(r=-0,22)$ (Jogi, y otros, 2013).

Se obtuvo una correlación alta, negativa, y altamente significativa, entre el peso del fruto de primera calidad y el número total de frutos por planta, para el total de genotipos evaluados $(\mathrm{r}$ $=-0,88 ; \mathrm{R} 2=0,78$ ) (figura 14), así como también para el grupo de pimiento cónico $(r=-0,73$; $R 2$ $=0,53$ ), mientras que para el grupo de pimiento cuadrado la correlación negativa fue muy baja y no significativa (tabla 17). Otros autores también hallaron una correlación alta, negativa, $\mathrm{y}$ altamente significativa entre estas variables $(r$ $=-0,68$ a $-0,82$ ) (Vikram, y otros, 2014; Bundela, 
Tabla 17

Coeficientes de correlación de Pearson entre peso del fruto de primera calidad y número total de frutos por planta

\begin{tabular}{lccc}
\hline Genotipos & $\begin{array}{c}\text { Coeficiente de } \\
\text { correlación }(\mathrm{r})\end{array}$ & Probabilidad $(\mathrm{p})$ & $\begin{array}{c}\text { Coeficiente de determinación de la } \\
\text { regresión lineal (R2) }\end{array}$ \\
\hline Total $(\mathrm{n}=27)$ & $-0,88$ & $* *$ & 0,78 \\
Cónico $(\mathrm{n}=12)$ & $-0,73$ & $* *$ & 0,53 \\
Cuadrado $(\mathrm{n}=13)$ & $-0,03$ & $\mathrm{~ns}$ & \\
\hline
\end{tabular}

Nota. $\mathrm{ns}=$ no significativo; ${ }^{*}=$ significativa $(\mathrm{p} \leq 0,05) ;{ }^{* *}=$ altamente significativa $(\mathrm{p} \leq 0,01)$.

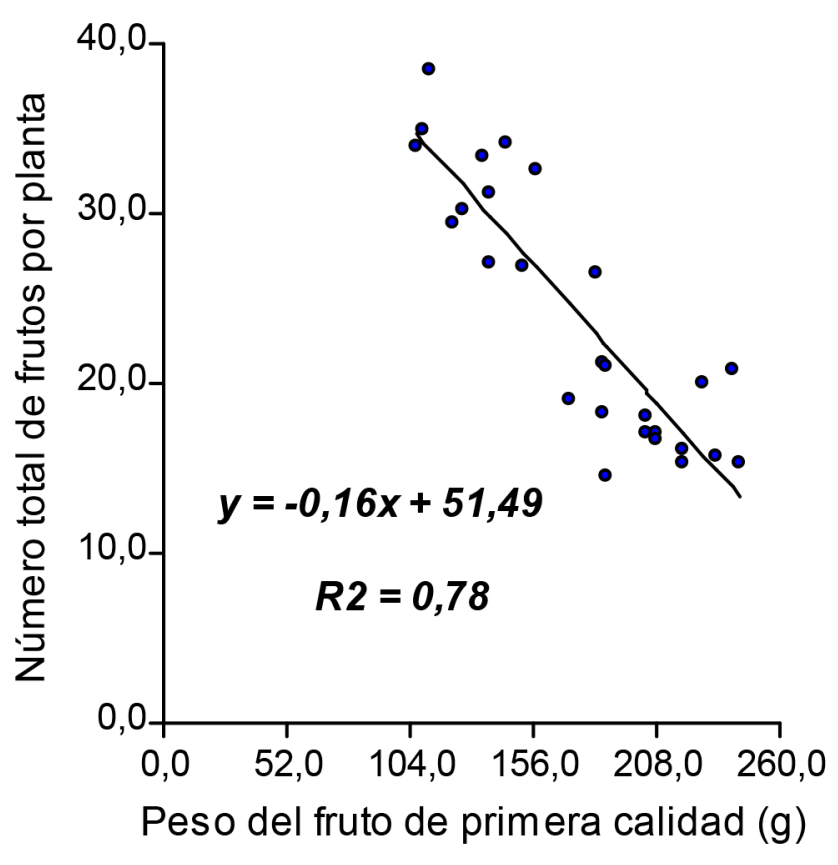

Figura 14. Regresión lineal del número total de frutos por planta versus el peso del fruto de primera calidad (g) para el total de genotipos evaluados $(n=27)$. y otros, 2018). En otros casos la correlación obtenida fue negativa y altamente significativa, pero baja ( $\mathrm{r}=-0,27$ a $-0,49)$ (Mathew, y otros, 2004; Yatung, y otros, 2014; Chakrabarty \& Islam, 2017). Y en otros estudios se halló una correlación baja, negativa y no significativa ( $\mathrm{r}$ $=-0,04$ a -0,42) (Madosa, y otros, 2008; Sasu, y otros, 2013; Cancaya, y otros, 2010; Bijalwan \& Mishra, 2016; Tembhurne \& Rao, 2013; Mamatha, Devaraju, Chand, \& Srinivasa, 2016; Dolkar, Madalageri, \& Manjunath, 2015; Aklilu, y otros, 2016; Rokib, y otros, 2016). Por otra parte, otros autores obtuvieron una correlación positiva y significativa, pero baja $(\mathrm{r}=0,20-0,28)$ (Pujar, y otros, 2017; Shweta, y otros, 2018). Y en otras investigaciones se encontró una correlación positiva, muy baja, y no significativa entre ambas variables $(\mathrm{r}=0,02-0,12)$ (Sharma, y otros, 2010; Pandit \& Adhikary, 2014).

Se encontró una correlación negativa y altamente significativa, aunque no fue alta, entre el peso del fruto de primera calidad y la longitud del fruto para el total de genotipos evaluados ( $\mathrm{r}$ $=-0,50$ ) (tabla 18), mientras que para el pimiento cuadrado fue positiva y no significativa ( $\mathrm{r}=$ 0,53), y únicamente para el pimiento cónico la correlación resultó alta, positiva, y altamente 
Tabla 18

Coeficientes de correlación de Pearson entre peso del fruto de primera calidad y longitud del fruto

\begin{tabular}{lccc}
\hline Genotipos & $\begin{array}{c}\text { Coeficiente de } \\
\text { correlación }(\mathrm{r})\end{array}$ & Probabilidad $(\mathrm{p})$ & $\begin{array}{c}\text { Coeficiente de determinación de la } \\
\text { regresión lineal (R2) }\end{array}$ \\
\hline Total $(\mathrm{n}=27)$ & $-0,50$ & $* *$ & 0,55 \\
Cónico $(\mathrm{n}=12)$ & 0,74 & $* *$ & \\
Cuadrado $(\mathrm{n}=13)$ & 0,53 & $\mathrm{~ns}$ & \\
\hline
\end{tabular}

Nota. $\mathrm{ns}=$ no significativo; ${ }^{*}=$ significativa $(\mathrm{p} \leq 0,05){ }^{* *}=$ altamente significativa $(\mathrm{p} \leq 0,01)$.

significativa $(\mathrm{r}=0,74 ; \mathrm{R} 2=0,55)$ (figura 15$)$. Otros autores también hallaron una correlación positiva, alta, y altamente significativa entre estas variables $(\mathrm{r}=0,83-0,89)$ (Bundela, y otros, 2018; Chakrabarty \& Islam, 2017). Por otra parte, en otras investigaciones se halló una correlación positiva, significativa o altamente significativa, pero no muy alta $(\mathrm{r}=0,24-0,56)$ (Sasu, y otros, 2013; Cancaya, y otros, 2010; Bijalwan \& Mishra, 2016; Vikram, y otros, 2014; Tembhurne \& Rao, 2013; Mamatha, y otros, 2016; Yatung, y otros, 2014; Shweta, y otros, 2018). En otros estudios se obtuvo una correlación positiva, baja, y no significativa entre ambas variables: en India ( $\mathrm{r}$ =0,05-0,36) (Sharma, y otros, 2010; Madosa, y otros, 2008; Pandit \& Adhikary, 2014; Misra, y otros, 2010; Mathew, y otros, 2004; Pujar, y otros, 2017; Aklilu, y otros, 2016; Rokib, y otros, 2016; Silva, y otros, 2013). Y en otra investigación, los valores obtenidos fueron $(r=0,23-0,24)$ (Ben-Chaim \& Paran, 2000). Y solamente en un caso se obtuvo una correlación negativa, baja y significativa entre estas variables $(r=-0,22)$ (Jogi, y otros, 2013).

Con respecto a la longitud del fruto y la altura de la planta, se halló una correlación positiva y altamente significativa, aunque no fue alta, para

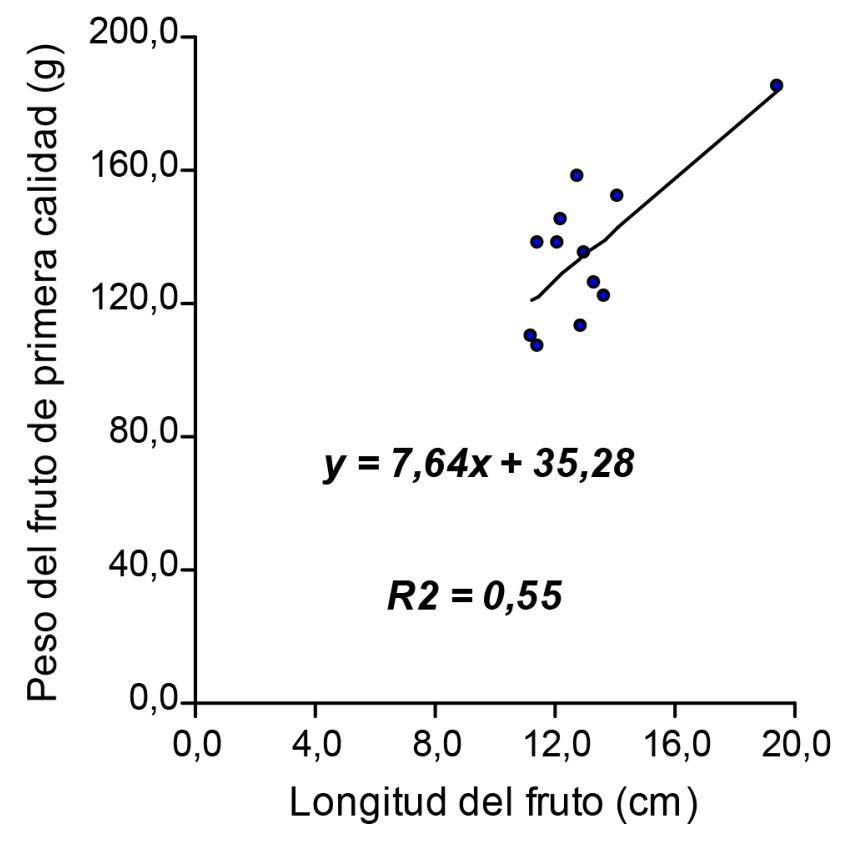

Figura 15. Regresión lineal del peso del fruto de primera calidad $(\mathrm{g})$ versus la longitud del fruto $(\mathrm{cm})$ para los pimientos cónicos $(n=12)$. 
el total de genotipos evaluados $(r=0,56)$ (tabla 19), mientras que para el pimiento cónico la correlación fue negativa y no significativa $(r=$ -0,56), y únicamente para el pimiento cuadrado la correlación fue alta, positiva, y altamente significativa $(r=0,74 ; R 2=0,55)$ (figura 16). En otros estudios se ha encontrado una correlación positiva, significativa o altamente significativa, pero no muy alta, entre ambas variables $(\mathrm{r}=$ 0,19 - 0,42) (Tembhurne \& Rao, 2013; Pujar, y otros, 2017; Shweta, y otros, 2018). Otros autores obtuvieron una correlación negativa, significativa $\mathrm{o}$ altamente significativa, pero no muy alta, entre estas variables $(r=-0,23$ a $-0,42$ ) (Chakrabarty \& Islam, 2017; Jogi, y otros, 2013). Otros investigadores obtuvieron una correlación positiva, baja, y no significativa entre ambas variables $(r=0,04-0,47)$ (Sharma, y otros, 2010; Mathew, y otros, 2004; Bundela, y otros, 2018; Aklilu, y otros, 2016; Yatung, y otros, 2014; Shumbulo, y otros, 2017). En otras investigaciones se obtuvo una correlación negativa, muy baja, y no significativa $(\mathrm{r}=-0,03$ a -0,22) (Cancaya, y otros, 2010; Bijalwan \& Mishra, 2016; Vikram, y otros, 2014; Mamatha, y otros, 2016; Vijaya, y otros, 2014; Misra, y otros, 2010; Rokib, y otros, 2016).

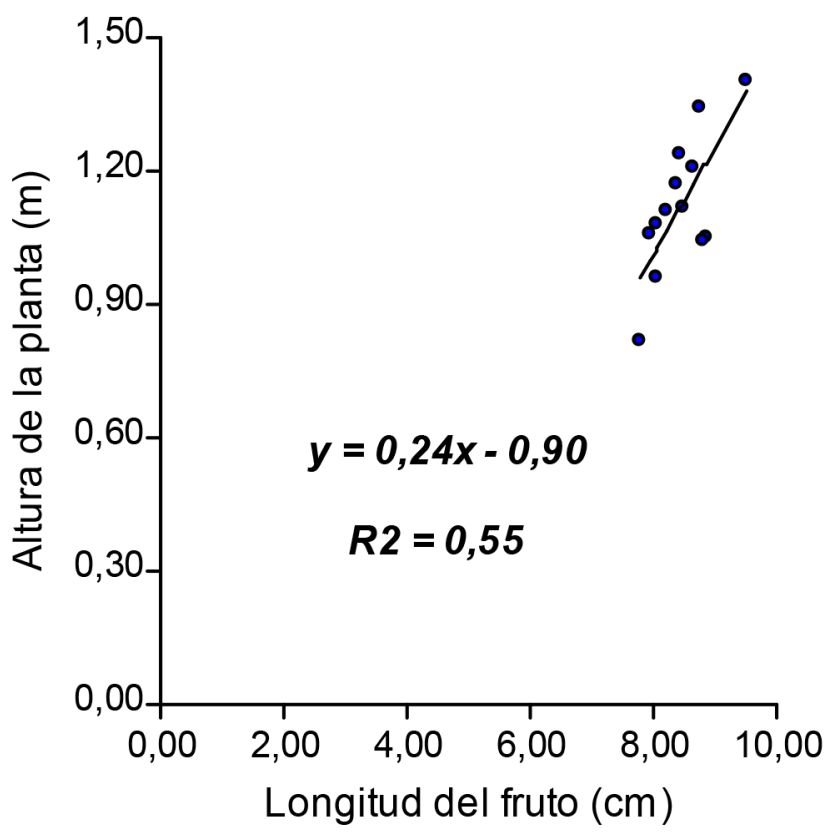

Figura 16. Regresión lineal de la altura de la planta (m) versus la longitud del fruto $(\mathrm{cm})$ para los pimientos cuadrados $(\mathrm{n}=13)$.

Tabla 19

Coeficientes de correlación de Pearson entre longitud del fruto y altura de la planta

\begin{tabular}{lccc}
\hline Genotipos & $\begin{array}{c}\text { Coeficiente de } \\
\text { correlación }(\mathrm{r})\end{array}$ & Probabilidad $(\mathrm{p})$ & $\begin{array}{c}\text { Coeficiente de determinación de la } \\
\text { regresión lineal (R2) }\end{array}$ \\
\hline Total $(\mathrm{n}=27)$ & 0,56 & $* *$ & \\
Cónico $(\mathrm{n}=12)$ & $-0,56$ & $\mathrm{~ns}$ & 0,55 \\
Cuadrado $(\mathrm{n}=13)$ & 0,74 & $* *$ & \\
\hline
\end{tabular}

Nota. $\mathrm{ns}=$ no significativo; ${ }^{*}=$ significativa $(\mathrm{p} \leq 0,05) ;{ }^{* *}=$ altamente significativa $(\mathrm{p} \leq 0,01)$. 
Se obtuvo una correlación positiva y altamente significativa, aunque no fue alta, entre el diámetro del tallo y la altura de la planta para el total de genotipos evaluados $(r=0,54)$ (tabla 20), mientras que para el pimiento cónico fue positiva y significativa, aunque tampoco fue alta $(r=0,63)$, y solamente para el pimiento cuadrado la correlación fue alta, positiva, y altamente significativa $(r=0,78 ; R 2=0,61)$ (figura 17). En otros estudios se encontró una correlación positiva y altamente significativa, pero baja, entre ambas variables $(r=0,30-0,40)$ (Cancaya, $y$ otros, 2010; Bundela, y otros, 2018; Pujar, y otros, 2017). Y otros autores hallaron una correlación positiva, baja, pero no significativa, entre estas variables $(r=0,23)$ (Shumbulo, y otros, 2017).

Sehalló una correlación positiva y significativa, aunque no fue alta, entre la altura de la planta y la edad al inicio de la cosecha para el total de genotipos evaluados $(r=0,42)$ (tabla 21), mientras que para el pimiento cuadrado fue positiva $y$ no significativa $(\mathrm{r}=0,30)$, y solamente para el pimiento cónico la correlación fue alta, positiva, $\mathrm{y}$ altamente significativa $(\mathrm{r}=0,70 ; \mathrm{R} 2=0,50)$ (figura 18). Otros investigadores encontraron una correlación positiva, y significativa o altamente significativa, pero no muy alta, entre estas

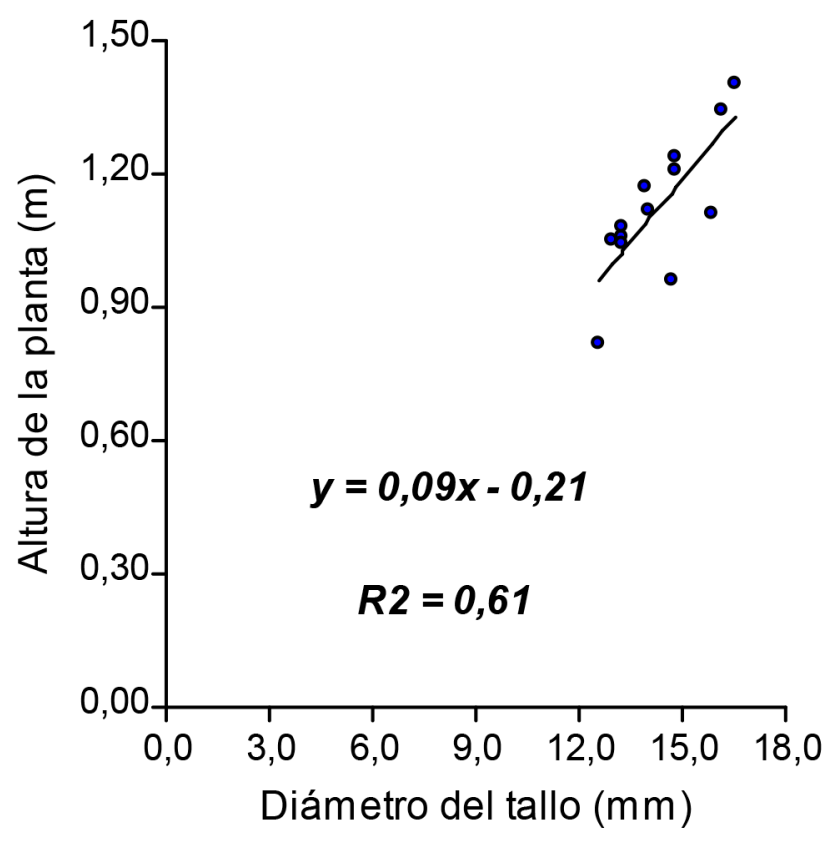

Figura 17. Regresión lineal de la altura de la planta (m) versus el diámetro del tallo $(\mathrm{mm})$ para los pimientos cuadrados $(n=13)$.

Tabla 20

Coeficientes de correlación de Pearson entre diámetro del tallo y altura de la planta

\begin{tabular}{lccc}
\hline Genotipos & $\begin{array}{c}\text { Coeficiente de } \\
\text { correlación }(\mathrm{r})\end{array}$ & Probabilidad $(\mathrm{p})$ & $\begin{array}{c}\text { Coeficiente de determinación de la } \\
\text { regresión lineal (R2) }\end{array}$ \\
\hline Total $(\mathrm{n}=27)$ & 0,54 & $* *$ & \\
Cónico $(\mathrm{n}=12)$ & 0,63 & $*$ & 0,61 \\
Cuadrado $(\mathrm{n}=13)$ & 0,78 & $* *$ & \\
\hline
\end{tabular}

Nota $. \mathrm{ns}=$ no significativo; ${ }^{*}=$ significativa $(\mathrm{p} \leq 0,05) ;{ }^{* *}=$ altamente significativa $(\mathrm{p} \leq 0,01)$. 
Tabla 21

Coeficientes de correlación de Pearson entre altura de la planta y edad al inicio de la cosecha

\begin{tabular}{lccc}
\hline Genotipos & $\begin{array}{c}\text { Coeficiente de } \\
\text { correlación }(\mathrm{r})\end{array}$ & Probabilidad $(\mathrm{p})$ & $\begin{array}{c}\text { Coeficiente de determinación de la } \\
\text { regresión lineal (R2) }\end{array}$ \\
\hline Total $(\mathrm{n}=27)$ & 0,42 & $*$ & 0,50 \\
Cónico $(\mathrm{n}=12)$ & 0,70 & $* *$ & \\
Cuadrado $(\mathrm{n}=13)$ & 0,30 & $\mathrm{~ns}$ & \\
\hline
\end{tabular}

Nota. $\mathrm{ns}=$ no significativo; ${ }^{*}=$ significativa $(\mathrm{p} \leq 0,05){ }^{* *}=$ altamente significativa $(\mathrm{p} \leq 0,01)$.

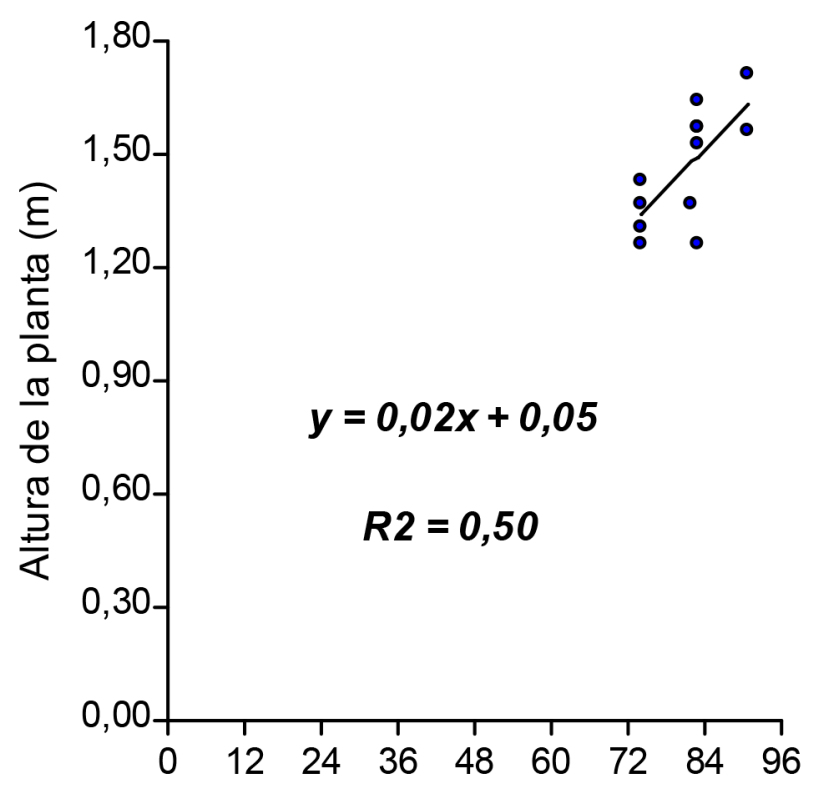

Edad al inicio de la cosecha (ddt)

Figura 18. Regresión lineal de la altura de la planta $(\mathrm{m})$ versus la edad al inicio de la cosecha $(\mathrm{ddt})$ para los pimientos cónicos $(\mathrm{n}=12)$. variables $(\mathrm{r}=0,37-0,60)$ (Vikram, y otros, 2014; Misra, y otros, 2010; Chakrabarty \& Islam, 2017). En otros estudios se obtuvo una correlación negativa y altamente significativa, pero no muy alta $(\mathrm{r}=-0,38$ a $-0,56)$ (Cancaya, y otros, 2010; Bijalwan \& Mishra, 2016; Mamatha, y otros, 2016). Otros autores hallaron una correlación positiva, baja y no significativa, entre ambas variables $(\mathrm{r}=0,03-0,39)$ (Sharma, y otros, 2010; Tembhurne \& Rao, 2013; Bundela, y otros, 2018). Y en un caso se obtuvo una correlación negativa, baja y no significativa entre esas variables $(\mathrm{r}=$ -0,13) (Rokib, y otros, 2016).

Se encontró una correlación positiva y altamente significativa, aunque no fue alta, entre el número total de frutos por planta y la longitud del fruto para el total de genotipos evaluados ( $\mathrm{r}$ $=0,56$ ) (tabla 22), mientras que para el pimiento cuadrado fue negativa y no significativa $(\mathrm{r}=$ $-0,31$ ), y únicamente para el pimiento cónico la correlación resultó alta, negativa, y altamente significativa $(r=-0,71 ; R 2=0,50)$ (figura 19). Unos autores obtuvieron una correlación negativa, altamente significativa, pero no muy alta, entre estas variables $(\mathrm{r}=-0,50$ a $-0,56)$ (Bundela, $\mathrm{y}$ otros, 2018; Chakrabarty \& Islam, 2017). En otros estudios se encontró una correlación positiva, significativa o altamente significativa, pero no 
Tabla 22

Coeficientes de correlación de Pearson entre número total de frutos por planta y longitud del fruto

\begin{tabular}{lccc}
\hline Genotipos & $\begin{array}{c}\text { Coeficiente de } \\
\text { correlación }(\mathrm{r})\end{array}$ & Probabilidad $(\mathrm{p})$ & $\begin{array}{c}\text { Coeficiente de determinación de la } \\
\text { regresión lineal (R2) }\end{array}$ \\
\hline Total $(\mathrm{n}=27)$ & 0,56 & $* *$ & 0,50 \\
Cónico $(\mathrm{n}=12)$ & $-0,71$ & $* *$ & \\
Cuadrado $(\mathrm{n}=13)$ & $-0,31$ & $\mathrm{~ns}$
\end{tabular}

Nota. $\mathrm{ns}=$ no significativo; ${ }^{*}=$ significativa $(\mathrm{p} \leq 0,05) ;{ }^{* *}=$ altamente significativa $(\mathrm{p} \leq 0,01)$.

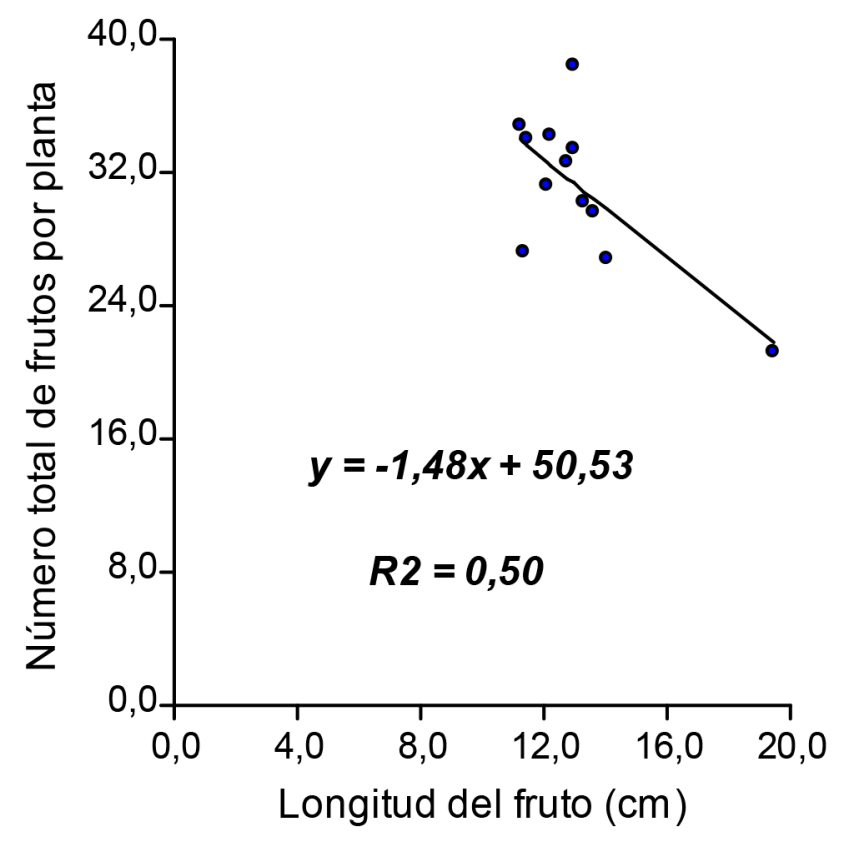

Figura 19. Regresión lineal del número total de frutos por planta versus la longitud del fruto $(\mathrm{cm})$ para los pimientos cónicos $(\mathrm{n}=12)$. muy alta entre ambas variables $(r=0,25-0,34)$ (Pandit \& Adhikary, 2014; Vijaya, y otros, 2014; Shweta, y otros, 2018). Otros investigadores hallaron una correlación positiva, baja, y no significativa entre ambas variables $(r=0,03-$ 0,25) (Sharma, y otros, 2010; Sasu, y otros, 2013; Mamatha, y otros, 2016; Aklilu, y otros, 2016; Rokib, y otros, 2016; Shumbulo, y otros, 2017). Por otra parte, en otros estudios se obtuvo una correlación negativa, baja y no significativa entre estas variables $(r=-0,01$ a $-0,22)$ (Madosa, y otros, 2008; Cancaya, y otros, 2010; Bijalwan \& Mishra, 2016; Vikram, y otros, 2014; Tembhurne \& Rao, 2013; Mathew, y otros, 2004; Pujar, y otros, 2017; Yatung, y otros, 2014).

Se obtuvo una correlación alta, positiva, y altamente significativa, entre el número de frutos de primera calidad por planta y el rendimiento comercial para el total de genotipos evaluados $(\mathrm{r}=0,78 ; \mathrm{R} 2=0,61)$ (figura 20), así como también para los grupos de pimiento cónico $(\mathrm{r}=0,75$; $\mathrm{R} 2$ $=0,56)$ o cuadrado $(\mathrm{r}=0,76 ; \mathrm{R} 2=0,58)($ tabla 23$)$.

En relación al número total de frutos por planta y el rendimiento comercial, se halló una correlación positiva y altamente significativa, aunque no fue alta, para el total de genotipos evaluados $(\mathrm{r}=0,51)$ (tabla 24$)$, mientras que para 


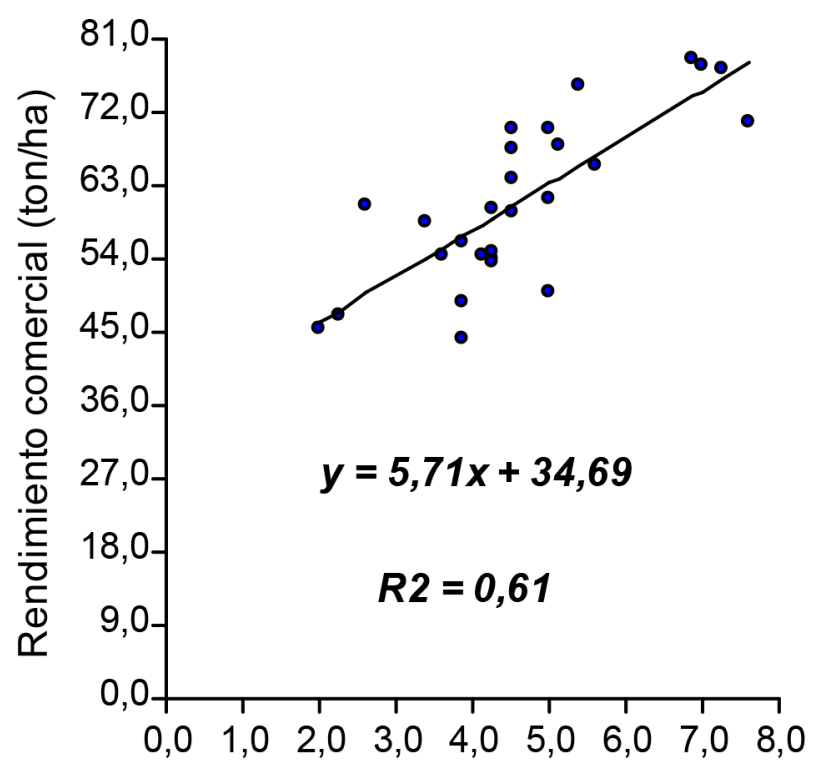

Número de frutos de primera calidad por planta

Figura 20. Regresión lineal del rendimiento comercial (ton/ha) versus el número de frutos de primera calidad por planta para el total de genotipos evaluados $(\mathrm{n}=27)$. el pimiento cónico la correlación fue positiva, pero no significativa $(\mathrm{r}=0,28)$, y únicamente para el pimiento cuadrado la correlación fue alta, positiva, $\mathrm{y}$ altamente significativa ( $\mathrm{r}=$ 0,72 ; R2 $=0,52$ ) (figura 21). Varios autores han obtenido una correlación alta, positiva, y significativa o altamente significativa, entre estas variables $(r=0,62-0,99)$ (Sharma, y otros, 2010; Cancaya, y otros, 2010; Madosa, y otros, 2008; Sasu, y otros, 2013; Pandit \& Adhikary, 2014; Bijalwan \& Mishra, 2016; Tembhurne \& Rao, 2013; Mamatha, y otros, 2016; Pujar, y otros, 2017; Dolkar, y otros, 2015; Rokib, y otros, 2016; Yatung, y otros, 2014; Shweta, y otros, 2018; Amit, Ahad, Kumar, \& Thakur, 2014). En otros estudios se encontró una correlación positiva, y significativa o altamente significativa, pero no tan alta, entre esas variables $(r=0,23-0,50)$ (Mathew, y otros, 2004; Bundela, y otros, 2018; Shumbulo, y otros, 2017). Y en un caso se obtuvo una correlación positiva, baja y no significativa entre estas variables $(r=0,29)$ (Vikram, y otros, 2014). Por otra parte, solamente en un estudio se halló una correlación negativa, baja, y altamente significativa $(\mathrm{r}=-0,02)$ (Chakrabarty \& Islam, 2017).

Tabla 23

Coeficientes de correlación de Pearson entre número total de frutos por planta y longitud del fruto

\begin{tabular}{lccc}
\hline Genotipos & $\begin{array}{c}\text { Coeficiente de } \\
\text { correlación }(\mathrm{r})\end{array}$ & Probabilidad $(\mathrm{p})$ & $\begin{array}{c}\text { Coeficiente de determinación de la } \\
\text { regresión lineal (R2) }\end{array}$ \\
\hline Total $(\mathrm{n}=27)$ & 0,78 & $* *$ & 0,61 \\
Cónico $(\mathrm{n}=12)$ & 0,75 & $* *$ & 0,56 \\
Cuadrado $(\mathrm{n}=13)$ & 0,76 & $* *$ & 0,58 \\
\hline
\end{tabular}

Nota. $\mathrm{ns}=$ no significativo; ${ }^{*}=$ significativa $(\mathrm{p} \leq 0,05) ;{ }^{* *}=$ altamente significativa $(\mathrm{p} \leq 0,01)$. 
Tabla 24

Coeficientes de correlación de Pearson entre número total de frutos por planta y rendimiento comercial

\begin{tabular}{lccc}
\hline Genotipos & $\begin{array}{c}\text { Coeficiente de } \\
\text { correlación }(\mathrm{r})\end{array}$ & Probabilidad $(\mathrm{p})$ & $\begin{array}{c}\text { Coeficiente de determinación de la } \\
\text { regresión lineal }(\mathrm{R} 2)\end{array}$ \\
\hline Total $(\mathrm{n}=27)$ & 0,51 & $* *$ & \\
Cónico $(\mathrm{n}=12)$ & 0,28 & ns & 0,52 \\
Cuadrado $(\mathrm{n}=13)$ & 0,72 & $* *$ &
\end{tabular}

Nota. $\mathrm{ns}=$ no significativo; ${ }^{*}=$ significativa $(\mathrm{p} \leq 0,05) ;{ }^{* *}=$ altamente significativa $(\mathrm{p} \leq 0,01)$.

\section{Conclusiones}

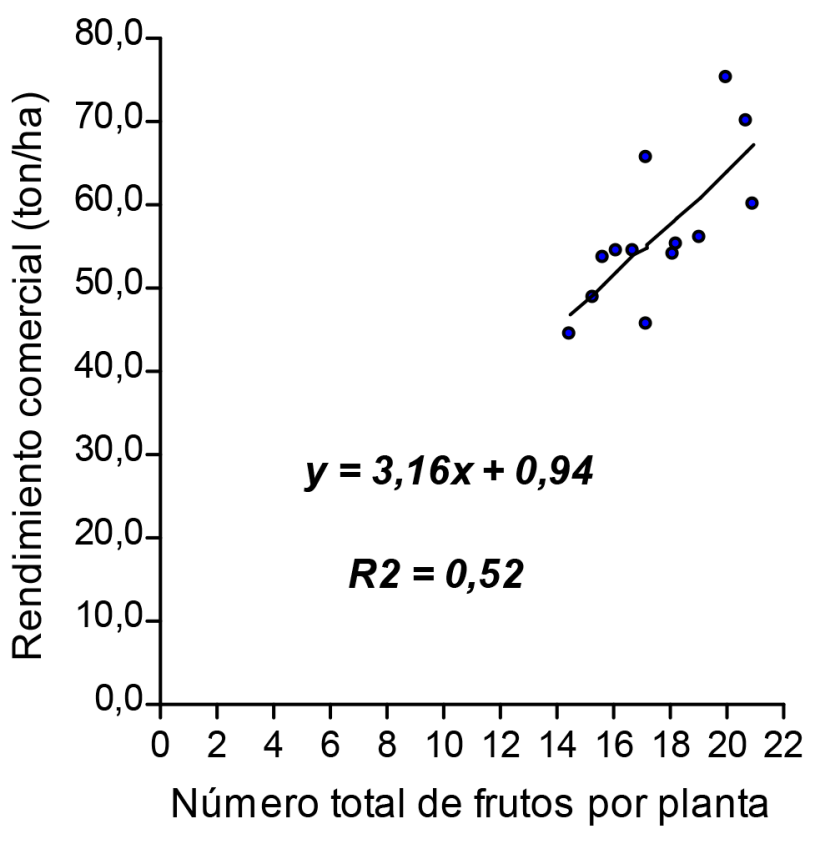

Figura 21. Regresión lineal del rendimiento comercial (ton/ha) versus el número total de frutos por planta para los pimientos cuadrados $(n=13)$.
Se presentaron 15 correlaciones de Pearson evaluadas en los 27 genotipos que fueron altas $(r \geq 0,69)$ y con significancia estadística $(p \leq 0,05)$, en cuyo caso se obtuvieron las regresiones lineales. Además, se obtuvieron otras seis correlaciones altas y con significancia estadística, según el tipo de pimiento (cónico o cuadrado).

Para las correlaciones de Pearson entre la altura de la planta y el área de la hoja, y entre el número de frutos de primera calidad por planta y el rendimiento comercial, se obtuvieron coeficientes de correlación altos y altamente significativos, tanto para el total de genotipos evaluados, como para los grupos según el tipo de pimiento (cónico o cuadrado). En todos los casos, la correlación fue positiva, es decir, se presentó una relación directamente proporcional entre los caracteres.

Sin embargo, para las demás correlaciones evaluadas, los resultados obtenidos fueron diferentes entre el total de genotipos evaluados y los grupos según el tipo de pimiento. De las 15 correlaciones que resultaron altas y con significancia estadística entre los 27 genotipos, en nueve de ellas las correlaciones fueron no significativas al considerar solamente los 12 genotipos de pimiento cónico, o los 13 de 
pimiento cuadrado, y en otras cuatro ocasiones la correlación fue menor a 0,69 o fue no significativa en al menos uno de los dos tipos de pimiento. A manera de ejemplo, existe una correlación alta y altamente significativa entre el peso del fruto $y$ el ancho del fruto en el pimiento cuadrado $\left(\mathrm{r}=0,71^{* *}\right)$, pero no en el pimiento cónico $(\mathrm{r}=$ $0,27 \mathrm{~ns}$ ); en otro caso, existe una correlación alta, negativa y altamente significativa entre el peso del fruto de primera calidad y el número total de frutos por planta en el pimiento cónico $(r=$ $\left.-0,73^{* *}\right)$, pero no en el pimiento cuadrado $(r=$ $-0,03 \mathrm{~ns})$. Y de manera interesante, se presentó una correlación alta y altamente significativa entre el número total de frutos por planta y el rendimiento comercial en el pimiento cuadrado $\left(\mathrm{r}=0,72^{* *}\right)$, pero no en el pimiento cónico $(\mathrm{r}=$ $0,28 \mathrm{~ns})$.

Por lo tanto, se concluye que el tipo de pimiento incluido en el análisis influye de manera importante en el resultado de las correlaciones entre variables. Para los fines de la selección genética y el fitomejoramiento en esta especie, este aspecto se debe tomar en cuenta al evaluar las asociaciones entre caracteres.

\section{Agradecimientos}

Los autores agradecen el financiamiento recibido por parte de la Universidad de Florida y de la Universidad de Costa Rica, para la realización de este trabajo. Asimismo, agradecen la colaboración de Jendry Portilla, Cristina Arguedas, Jorge Díaz y Julio Vega en el trabajo de campo, y de Mario Monge en la revisión de la traducción del resumen al idioma inglés.

\section{Referencias}

Aklilu, S., Abebie, B., Wogari, D., \& Wolde, A. T. (2016). Genetic variability and association of characters in ethiopian hot pepper (Capsicum annuum L.) landraces. Journal of Agricultural Sciences, 61(1), 19-36.
Amit, K., Ahad, I., Kumar, V., \& Thakur, S. (2014). Genetic variability and correlation studies for growth and yield characters in chilli (Capsicum annuum L.). Journal of Spices $\mathcal{E}$ Aromatic Crops, 23(2), 170-177.

Baseerat, A., Khan, S. H., Mushtaq, F., Hussain, K., \& Nabi, A. (2013). Variability and correlation studies in sweet pepper (Capsicum annuum L.). Progressive Horticulture, 45(1), 209-213.

Ben-Chaim, A., \& Paran, I. (2000). Genetic analysis of quantitative traits in pepper (Capsicum annuum). Journal of the American Society for Horticultural Science, 125(1), 66-70.

Bharadwaj, D. N., Singh, H., \& Yadav, R. K. (2007). Genetic variability and association of component characters for yield in chilli (Capsicum annuum L.). Progressive Agriculture, 7(1-2), 72-74.

Bijalwan, P., \& Mishra, A. C. (2016). Correlation and path coefficient analysis in chilli (Capsicum annuum L.) for yield and yield attributing traits. International Journal of Science and Research, 5(3), 1589-1592.

Bundela, M. K., Pant, S. C., Madhuri, \& Singh, K. (2018). Correlation and path coefficient analysis in chilli (Capsicum annuum L.) for yield and yield attributing traits. International Journal of Current Microbiology and Applied Sciences, 7(11), 65-70.

Cancaya, S., Balkaya, A., \& Karaagac, O. (2010). Canonical correlation analysis for the determination of relationships between plant characters and yield components in red pepper [Capsicum annuum L. var. conoides (Mill.) Irish] genotypes. Spanish Journal of Agricultural Research, 8(1), 67-73.

Castilla, N. (2005). Invernaderos de plástico: tecnología y manejo. España: Mundi Prensa. 
Chakrabarty, S., \& Islam, A. K. (2017). Selection criteria for improving yield in chili (Capsicum annuum). Advances in Agriculture, 2017, 1-9.

Diwaker, K., Vijay, B., Rangare, S. B., \& Devi, S. (2012). Genetic variability, heritability and correlation studies in chilli (Capsicum annuum L.). HortFlora Research Spectrum, 1(3), 248-252.

Dolkar, R., Madalageri, M. B., \& Manjunath, G. (2015). Correlation and path analysis for growth, earliness, yield and quality parameters in chilli (Capsicum annuum L.). HortFlora Research Spectrum, 4(3), 268-272.

Gil-Vásquez, I., Sánchez del Castillo, F., \& Miranda-Velásquez, I. (2003). Producción de jitomate en hidroponía bajo invernadero. Chapingo, México: Serie de publicaciones Agibot.

Gupta, A. M., Daljeet, S., \& Ajay, K. (2009). Genetic variability, genetic advance and correlation in chilli (Capsicum annuum). Indian Journal of Agricultural Sciences, 79(3), 221-223.

IPGRI. (1995). Descriptores para Capsicum (Capsicum spp.). Roma, Italia: Instituto Internacional de Recursos Fitogenéticos.

Jiménez, U., Campos, H., Vicente, J., Marín, S., Barrantes, L., \& Carrillo, M. (2007). Agrocadena regional: cultivo del chile dulce. Grecia, Alajuela, Costa Rica: Dirección Regional Central Occidental, Ministerio de Agricultura y Ganadería.

Jogi, M. Y., Madalageri, M. B., Ganiger, V. M., Bhuvaneswari, G., Patil, H. B., \& Kotikal, Y. K. (2013). Character association and path analysis studies in green chilli (Capsicum annuum L.). International Journal of Agriculturas Sciences, 9(2), 547-550.

Madosa, E., Ciulca, S., Velicevici, G., Avadanei, C., Sasu, L., Cioroga, A., \& Friskan, I. (2008). Study of correlations between component characters of production capacity of sweet pepper (Capsicum annuum L. var. grossum). Bulletin UASVM, Horticulture, 65(1), 90-94.

Mamatha, A., Devaraju, Chand, U. P., \& Srinivasa, V. (2016). Genetic correlation and path coefficient analysis in chilli (Capsicum annuum L.) genotypes under hill zone of Karnataka. The Bioscan, 11(3), 1995-1998.

Marín, F. (2009). Programa general de la agricultura protegida y sus aplicaciones en Costa Rica. San José, Costa Rica: Programa Nacional Sectorial de Producción Agrícola Bajo Ambientes Protegidos, Ministerio de Agricultura y Ganadería.

Mathew, D., Doijode, S. D., \& Reddy, K. M. (2004). Correlation and path coefficient analysis in five species of Capsicum. Capsicum and Eggplant Newsletter, 23, 57-60.

Misra, S., Lal, R. K., Darokar, M. P., \& Khanuja, S. P. (2010). Genetic associations and pathcoefficient analysis of the economic traits in the chili (Capsicum annuum L.). Electronic Journal of Plant Breeding, 1(3), 346-350.

Pandit, M. K., \& Adhikary, S. (2014). Variability and heritability estimates in some reproductive characters and yield in chilli (Capsicum annuum L.). International Journal of Plant $\mathcal{E}$ Soil Science, 3(7), 845-853.

Pujar, U. U., Tirakannanavar, S., Jagadeesha, R. C., Gasti, V. D., \& Sandhyarani, N. (2017). Genetic variability, heritability, correlation and path analysis in chilli (Capsicum annuum L.). International Journal of Pure $\mathcal{E}$ Applied Bioscience, 5(5), 579-586.

Rokib, H., Matin, A., Nazmul, A., Abul, B., \& Mahmudul, H. A. (2016). Genetic association analysis and selection indices for yield attributing traits in available chilli (Capsicum annuum L.) genotypes. Molecular Plant Breeding, 7(19), 1-9. 
Sasu, L., Madosa, E., Velicevici, G., Ciulca, S., Avadanei, C., \& Gorinoiu, G. (2013). Studies regarding correlations between the main morphological traits in a collection of bell pepper (Capsicum annuum var. grossum) local landraces. Journal of Horticulture, Forestry and Biotechnology, 17(2), 285-289.

Sharma, V. K., Semwal, C. S., \& Uniyal, S. P. (2010). Genetic variability and character association analysis in bell pepper (Capsicum annuum L.). Journal of Horticulture and Forestry, 2(3), 58-65.

Shumbulo, A., Nigussie, M., \& Alamerew, S. (2017). Correlation and path coefficient analysis of hot pepper (Capsicum annuum L.) genotypes for yield and its components in Ethiopia. Advances in Crop Science and Technology, 5(3), 1-5.

Shweta, Basavarajappa, H. R., Satish, D., Jagadeesha, R. C., Hanachinmani, C. N., \& Dileepkumar, A. M. (2018). Genetic correlation and path coefficient analysis in chilli (Capsicum annuum L.) genotypes for growth and yield contributing traits. Journal of Pharmacognosy and Phytochemistry, 7(2), 1312-1315.

Silva, A. R., Nascimento, M., Cecon, P. R., Sapucay, M. J., Rêgo, E. R., \& Barbosa, L. A. (2013). Path analysis in multicollinearity for fruit traits of pepper. IDESIA, 31(2), 55-60.
Tembhurne, B. V., \& Rao, S. K. (2013). Stability analysis in chilli (Capsicum annuum L.). Journal of Spices and Aromatic Crops, 22(2), 154-164.

Vijaya, H. M., Gowda, A. P., \& Nehru, S. D. (2014). Genetic variability, correlation coefficient and path analysis in chilli (Capsicum annuum L.) genotypes. Research in Environment and Life Sciences, 7(3), 175-178.

Vikram, A., Warshamana, I., \& Gupta, M. (2014). Genetic correlation and path coefficient studies on yield and biochemical traits in chilli (Capsicum annuum L.). International Journal of Farm Sciences, 4(2), 70-75.

Wittwer, S., \& Castilla, N. (1995). Protected cultivation of horticulture crops worldwide. Hort Technology, 5(1), 6-22.

Yatung, T., Dubey, R. K., Singh, V., Upadhyay, G., \& Pandey, A. K. (2014). Selection parameters for fruit yield and related traits in chilli (Capsicum annuum L.). Bangladesh Journal of Botany, 43(3), 283-291.

Zúñiga-Estrada, L., Martínez-Hernández, J. d., Baca-Castillo, G. A., Martínez-Garza, A., Tirado-Torres, J. L., \& Kohashi-Shibata, J. (2004). Producción de chile pimiento en dos sistemas de riego bajo condiciones hidropónicas. Agrociencia, 38(2), 207-218.

Recibido: 14 de enero de 2019 Aceptado: 21 de noviembre de 2019 